\title{
The Concentration Function Problem for Locally Compact Groups Revisited: Nondissipating Space-Time Random Walks, $\tau$-Decomposable Laws, and Their Continuous Time Analogues
}

\author{
Wilfried Hazod \\ Faculty of Mathematics, TU Dortmund University, D-44221 Dortmund, Germany \\ Correspondence should be addressed to Wilfried Hazod; wilfried.hazod@mathematik.tu-dortmund.de
}

Received 28 November 2012; Accepted 15 July 2013

Academic Editor: Ralf Meyer

Copyright (c) 2013 Wilfried Hazod. This is an open access article distributed under the Creative Commons Attribution License, which permits unrestricted use, distribution, and reproduction in any medium, provided the original work is properly cited.

The concentration function problem for locally compact groups is concerned with the structure of groups admitting adapted nondissipating random walks. It is closely related to discrete relatively compact $\mathrm{M}$ - or skew convolution semigroups and corresponding space-time random walks, and to $\tau$-decomposable laws, respectively, where $\tau$ denotes an automorphism. Analogous results are obtained in the case of continuous time: nondissipating Lévy processes are related to relatively compact distributions of generalized Ornstein-Uhlenbeck processes and corresponding space-time processes and to $T$-decomposable laws, respectively with $T=\left(\tau_{t}\right)$ denoting a continuous group of automorphisms acting as contracting mod. a compact subgroup.

\section{Introduction}

Let $\mathbb{G}$ be a locally compact group, $\lambda \in \mathscr{M}^{1}(\mathbb{G})$ an (w.l.o.g.) adapted probability measure. $\lambda$, more precisely, the random walk $\left\{\lambda^{k}\right\}_{k>0}$, is called nondissipating (also nonscattering) if for some compact subset $C \subseteq \mathbb{G}$ the (right) concentration functions $f_{\lambda^{k}}(C):=\sup _{x \in \mathbb{G}} \lambda^{k}(C x)$ fail to converge to 0 , with time $k \rightarrow \infty$. To avoid trivialities, throughout $\mathbb{G}$ is supposed to be noncompact, else any random walk would be nondissipating.

If the random walk is nondissipating, $\left\{\lambda^{k}\right\}_{k \geq 0}$ is relatively (right) shift compact, equivalently, $\left\{\lambda^{k} * \tilde{\lambda}^{k}\right\}_{k \geq 0}$ is relatively compact [1].

Furthermore, if $N=N_{\lambda}$ denotes the smallest closed normal subgroup of $G$ containing the support supp $\lambda * \widetilde{\lambda}$, then $\mathbb{G} / N \cong \mathbb{Z}$. Hence there exists $x \in \mathbb{G}$ such that $\lambda=\nu * \varepsilon_{x}$ with supp $v \subseteq N$.

Denoting the restriction of the inner automorphism $i_{x}$ : $z \mapsto x z x^{-1}$ to $N$ by $\tau:=\left.i_{x}\right|_{N}$, we obtain $G \cong N \rtimes_{\tau} \mathbb{Z}$, and $\lambda$ is represented as $\lambda=\nu(1) \otimes \varepsilon_{1}$, with $\nu=\nu(1) \in \mathscr{M}^{1}(N)$ hence the random walk is representable as $\lambda^{k}=\nu(k) \otimes \varepsilon_{k}$, for all $k \in \mathbb{Z}_{+}\left(\right.$with $\nu(k) \in \mathscr{M}^{1}(N), \nu(0):=\varepsilon_{0}$.)
The existence of nondissipating adapted random walks has a strong impact on the structure of $\mathbb{G}$ : it is shown in [1] that $N=C_{K}(\tau)$, where $K$ is a compact $\tau$-invariant subgroup of $N$ and $C_{\{\}\}}(\tau)=: C(\tau):=\left\{x \in N: \tau^{n}(x) \rightarrow\right.$ $e\}$ (for $K=\{e\}$ ), respectively, $C_{K}(\tau):=\{x \in N$ : $\left.\tau^{n}(x) \rightarrow e \bmod K\right\}$ denote the contractible, respectively, the $K$-contractible subgroups. As easily seen, for a random walk $\left\{\lambda^{k}=\nu(k) \otimes \varepsilon_{k}\right\} \subseteq \mathscr{M}^{1}\left(N \rtimes_{\tau} \mathbb{Z}\right), \nu(k)$ is representable as $\nu(k)=\star_{j=0}^{k-1} \tau^{j}(\nu), k \in \mathbb{N},(“ \star$ ” denoting convolution on $N$ ) and hence satisfies the relation $\nu(k+\ell)=\nu(k) \star \tau^{k}(\nu(\ell)), k, \ell \in$ $\mathbb{Z}_{+}$. We call a sequence $\{\nu(k)\}_{k \in \mathbb{Z}_{+}}$satisfying such a relation "discrete time M-(or Mehler-)-semigroup" (in fact, in analogy to the continuous time case, the (discrete) semigroup of transition kernels is called discrete Mehler semigroup.) This $\mathrm{M}$-(convolution)-semigroup is also called skew convolution semigroup or $\tau$-semigroup. Moreover, if $\left\{\lambda^{k}\right\}$ is nondissipating, the M-semigroup $\{\nu(k)\}$ is relatively compact, a crucial property in the sequel. Conversely, discrete M-semigroups $\{\nu(k)\} \subseteq \mathscr{M}^{1}(N)$ define space-time random walks $\left\{\lambda^{k}=\nu(k) \otimes\right.$ $\left.\varepsilon_{k}\right\}$ on the space-time building $\mathbb{G}=N \rtimes_{\tau} \mathbb{Z}$. So, nondissipating adapted random walks (on $\mathbb{G}$ ) correspond in a 1-1-manner to relatively compact $\mathrm{M}$-semigroups (on N), cf. Theorem 6 later. 
We furthermore show that $\rho:=\lim _{k \rightarrow \infty} \nu(k) \star \omega_{K}$ exists and is $\tau$-decomposable, that is, $\rho=\nu(k) * \tau^{k}(\rho)$ with relatively compact M-semigroups of cofactors $\{\nu(k)\}_{k \in \mathbb{Z}_{+}}$; furthermore, $\rho$ is right $K$-invariant. Conversely, for any such measure $\rho$ with cofactors $\{\nu(k)\}$, we have $\lim \nu(k) \star \omega_{K}=\rho$. Thus $\tau$-decomposable measures $\rho$, respectively, and the cofactors $\{\nu(k)\}$ generate nondissipating random walks and vice versa.

The first part of the paper may be considered as an overview of more or less known results around the concentration function problem and its relations to $\mathrm{M}$-semigroups and $\tau$-decomposability. These results are needed as motivation and as a tool box for proofs in the second part which is concerned with the continuous time analogues. The random walk is replaced by a continuous convolution semigroup $\left\{\lambda_{t}\right\}_{t \in \mathbb{R}_{+}}$, the distributions of a Lévy process. At first (Propositions 12 and 13), we show that $\left\{\lambda_{t}\right\}_{t \in \mathbb{R}_{+}}$is nondissipating and adapted iff some (hence all) skeleton random walk $\left\{\lambda_{t_{0}}^{k}=\lambda_{t_{0} k}\right\}_{k \in \mathbb{Z}_{+}}$is nondissipating; it is adapted on $\mathbb{G}_{t_{0}}$, the group generated by $\operatorname{supp} \lambda_{t_{0}}$. Furthermore, we show that the subgroup $N:=N_{\lambda_{t_{0}}}$ is independent of $t_{0}>0$ and is a normal subgroup in $\mathbb{G}$ (not only within the group $\mathbb{G}_{t_{0}}$ ), and we observe $\mathbb{G}=\bigcup_{t} \mathbb{G}_{t}$. Thus the results for discrete times apply easily to the continuous time setup. Furthermore, $\mathbb{G} / N \cong \mathbb{R}$, and there exists a continuous one-parameter group $(x(t))_{t \in \mathbb{R}}$ such that with $T=$ $\left(\tau_{t}:=\left.i_{x(t)}\right|_{N}\right) \subseteq \operatorname{Aut}(N), \mathbb{G} \cong N \rtimes_{T} \mathbb{R}$. Moreover, in that case, there exists a homogeneous group (i.e., a contractible Lie group) $C$ and a compact $T$-invariant subgroup $K$, such that $N=C_{K}(T)=C \rtimes_{\beta} K, \beta \in \operatorname{Hom}(K, \operatorname{Aut}(C))$.

Theorems 20 and 22 contain the continuous time analogues of Theorem 6, in particular, also a characterization of relative compactness of M-semigroups in terms of logarithmic, respectively, first order moments of the corresponding Lévy measure (on $N$ resp., $\mathbb{G}$.)

One of the aims of the paper is to point out that different branches of investigations lead to the same or equivalent objects: (1) nondissipating random walks, respectively, continuous convolution semigroups, (2) $\tau$-decomposable laws and the subclasses of (semi-) stable and self-decomposable laws, and (3) (relatively compact) skew (discrete or continuous) convolution semigroups. While in the past in the cases (2) and (3) most investigations were concerned with continuous time models, the emphasis in the first case was on discrete time models.

All these investigations-for discrete and continuous times-are related to the structure of contractible, respectively, $K$-contractible subgroups $C(\tau)$, respectively, $C_{K}(\tau)$ of locally compact groups. See for example, [2-5], [6, Ch. III], $[1,7-15]$, and the literature mentioned there.

For the history of the concentration function problem for random walks on locally compact groups the reader is referred to the survey of Jaworski [1] showing previous developments and a recent state of investigations: beginning with the pioneer works [16-20] to the investigations $[1,11,13]$.

Continuous time models (for the cases (2) and (3)) had been investigated in the past in different papers. Beginning with the pioneer work [21] (with slightly different representations) to $[22,23],[6, \$ 2.14]$. See also [24] for Mehler hemigroups and embedding of discrete time models into continuous time ones. In [22] the reader will find some more hints to the literature, in particular also to vector spaces, beyond the locally compact group case.

\section{Discrete Time: Nondissipating Random Walks}

Recall the following notations: $\mathbb{G}$ denotes a locally compact group with unit $e$. $\mathfrak{U}_{\mathbb{G}}$ denotes the filter basis of Borel neighbourhoods of the unit. For $\lambda \in \mathscr{M}^{1}(\mathbb{G})$, the set of probabilities, let $\tilde{\lambda}$ be the image of $\lambda$ under the inverse mapping $x \mapsto x^{-1}$. * Denotes convolution on $\mathbb{G}, \lambda^{k}$ the $k$ th convolution power, $\lambda^{0}:=\varepsilon_{e}$, where $\varepsilon_{x}$ denotes the point measure in $x \in \mathbb{G}$. w.l.o.g. $\lambda$ is supposed to be adapted; that is, $\mathbb{G}$ is the closed group generated by the support supp $\lambda . N=$ $N_{\lambda}$ denotes the smallest closed normal subgroup containing supp $\lambda * \widetilde{\lambda}$. The concentration function of the random walk $\left\{\lambda^{k}\right\}_{k \in \mathbb{Z}_{+}}$is defined as $\mathbb{Z}_{+} \ni k \mapsto f_{\lambda^{k}}(C):=\sup _{x \in \mathbb{G}} \lambda^{k}(C x)$ for compact $C \subseteq \mathbb{G}$. The probability measure $\lambda$, or more precisely, the random walk $\left\{\lambda^{k}\right\}$ is called nondissipating if $f_{\lambda^{k}}(C)$ fails to converge to 0 with $k \rightarrow \infty$, for some compact $C$. Recall that we defined afore for $\tau \in \operatorname{Aut}(N)$ and a compact $\tau$-invariant subgroup $K: C_{K}(\tau):=\left\{x \in N: \tau^{k}(x) \rightarrow e \bmod K\right\}$ and $C(\tau)=C_{\{e\}}(\tau)=\left\{x: \tau^{k}(x) \rightarrow e\right\} . \tau \in \operatorname{Aut}(\mathbb{G})$ is contracting if $C(\tau)=\mathbb{G}$. And if a contracting automorphism exists, $\mathbb{G}$ is called contractible. Analogously we define $K$-contracting automorphisms, respectively, $K$-contractible groups, where $K$ denotes a compact $\tau$-invariant subgroup. LIM will throughout denote the set of accumulation points, and $A^{-}$denotes the closure of a set $A$.

We collect some properties of adapted random walks:

Fact 1. (a) $\left\{\lambda^{k}\right\}$ is nondissipating iff $\left\{\lambda^{k}\right\}$ is relatively (right) shift compact; that is, for some $x_{k} \in \mathbb{G},\left\{\lambda^{k} * \varepsilon_{x_{k}^{-1}}\right\}$ is relatively compact. Equivalently, iff $\left\{\lambda^{k} * \tilde{\lambda}^{k}\right\}$ is relatively compact. Indeed, then $\lim _{k} \lambda^{k} * \tilde{\lambda}^{k}$ exists.

In that case, if $\mathbb{G}$ is noncompact, the following assertions (b)-(f) hold:

(b) $\mathbb{G} / N \cong \mathbb{Z}$, hence the shifts can be chosen as $x_{k}=x^{k}$ for some $x \in \mathbb{G} \backslash N$.

(c) The restriction to $N$ of the inner automorphism $\tau:=$ $\left.i_{x}\right|_{N}, z \mapsto x z x^{-1}$, is considered as automorphism of $N$, and hence $\mathbb{G}$ has a canonical representation $\mathbb{G}=N \rtimes_{\tau} \mathbb{Z}$ (with product $(g, k)(h, \ell)=\left(g \tau^{k}(h), k+\ell\right)$ for $g, h \in N, k, \ell \in \mathbb{Z}$, where $x=(e, 1)$.)

(d) There exists a compact subgroup $K \subseteq N$ such that $N=C_{K}(\tau)$. If $N$ is compact then $N=K$.

(e) $\lambda$ and $\nu:=\lambda * \varepsilon_{x^{-1}}$ are representable as $\lambda=\nu \otimes$ $\varepsilon_{1} \in \mathscr{M}^{1}\left(N \rtimes_{\tau} \mathbb{Z}\right), v=: \nu(1)$ is identified with a probability of $\mathscr{M}^{1}(N)$. Hence by induction, $\lambda^{k}=\nu(k) \otimes \varepsilon_{k}, k \in \mathbb{Z}_{+}$, with $\nu(0)=\varepsilon_{e}, \nu(1)=\nu, \nu(k)=\star_{j=0}^{k-1} \tau^{j}(\nu)=: \nu \star$ $\tau(\nu) \star \cdots \star \tau^{k-1}(\nu) \in \mathscr{M}^{1}(N)$, and $x$ is identified with $(e, 1)$. Furthermore, $\{\nu(k)\}_{k \in \mathbb{Z}_{+}}$is relatively compact. (Indeed, as $\lambda^{k} *$ $\tilde{\lambda}^{k}$ is convergent, $\lim \nu(k) \star \widetilde{\nu(k)}$ exists.) To distinguish, in the sequel “*” will denote convolution on $\mathbb{G}$ while convolution 
on $N$ is denoted by “*”. (Thus we have $\left(\mu \otimes \varepsilon_{k}\right) *\left(\nu \otimes \varepsilon_{\ell}\right)=$ $\left.\left(\mu \star \tau^{k}(\nu)\right) \otimes \varepsilon_{k+\ell}.\right)$

(f) Put $L:=\bigcup_{k \in \mathbb{Z}_{+}} \operatorname{supp} v(k)$. Then $N$ is the smallest closed $\tau$-invariant normal subgroup of $\mathbb{G}$ containing $L$. (The closed group generated by $L$ may be smaller than $N$.)

[(a) See [1, Corollary 3.2] and the literature mentioned there. In particular, [25], [12, Theorem 1]. For the convergence of $\lambda^{k} * \tilde{\lambda}^{k}$, see $[26,27]$.

(b), (c) See [1, Theorem 3.4], [25]. (d) See [1, Theorems 3.5 and 3.9]. If $N=C_{K}(\tau)$ is compact, then, as $\tau$ is compactly contracting, $N=K$ follows.

(e) The representation of $\{v(k)\}$ is immediately verified, and relative compactness follows by [1, Theorem 3.9].

(f) $\lambda$ is adapted, hence $\mathbb{G}$ is generated by $\operatorname{supp} v(1) \otimes\{1\}$. $N \triangleleft \mathbb{G}$, hence $N$ is $\tau$-invariant and $N \supseteq L$. By definition, $N$ is the smallest closed normal subgroup with these properties. It is easily shown by examples that $N$ may be larger than the group generated by $L . \rrbracket$

As already mentioned, $\mathbb{G}$ is always supposed to be noncompact, else any random walk would be nondissipating. If $N$ is compact then (by Fact $1(\mathrm{~d})$ ), $N=K$ and any "spacetime" random walk $\left\{\lambda^{k}=\nu(k) \otimes \varepsilon_{k}\right\}, \nu(k) \in \mathscr{M}^{1}(N)$, is nondissipating.

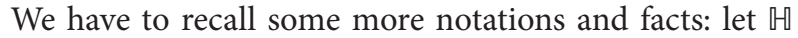
be a locally compact group. A function $f: \mathbb{W} \rightarrow \mathbb{R}_{+}$ is subadditive (resp., a "gauge") if $f(x y) \leq f(x)+f(y)$ (resp., $\leq C+f(x)+f(y)$ for some constant $C \geq 0)$. $f$ is submultiplicative if $f(x y) \leq f(x) f(y)$. As immediately seen, if $f$ is subadditive (and $\geq 0$ ), then $g:=1+f$ is submultiplicative (and $\geq 1$ ), and if $h$ is submultiplicative (and $\geq 1$ ), then $\log (h)$ is subadditive (and $\geq 0$ ). Thus $\log (1+f)$ is subadditive and $1+\log (1+f)$ is submultiplicative. We will always assume tacitly that $f$ is Borel measurable. Therefore, $f$ is locally bounded (see [28, Proposition 1]), hence there exist $\epsilon>0$ such that $U_{f, \epsilon}:=\{f<\epsilon\} \in \mathfrak{U}_{\mathbb{}}$. To any $f$ we fix such an $\epsilon$ (and a neighbourhood $U \subseteq U_{f, \epsilon}$ ).

Let $\mathbb{U}$ be compactly generated, $\mathbb{U}=\bigcup_{n \geq 0} V^{n}$ for some symmetric compact $V \subseteq \mathfrak{U}_{\mathfrak{H}}$. Then we define a subadditive function $\delta_{V}: x \mapsto \inf \left\{n \in \mathbb{Z}_{+}: x \in V^{n}\right\}$. According to [28, Proposition 1], any gauge, in particular any subadditive function $f$, is dominated by $\delta_{V}$, that is,

$$
f \leq A \delta_{V}+B \text { for some constants } A>0, B \geq 0 .
$$

Definition 1. (a) Let $\mathbb{W}=N=C_{K}(\tau)$. A subadditive function $f \geq 0$ is called $\tau$-function (cf. [1, Definition 2.6]) if for some $p \in \mathbb{N}, 0<\delta<1$, we have $f\left(\tau^{p}(x)\right)=\delta \cdot f(x), x \in \mathbb{M}$. $f$ is a regular $\tau$-function if in addition $f\left(x^{-1}\right)=f(x),\{f \leq \alpha\}$ is relatively compact for some (hence all) $\alpha>0$ and $f(x)=0$ iff $x \in K$. In fact, $f$ is continuous and $K$-bi-invariant (see [1, Propositions 2.8 and 2.10].)

Let $\mu \in \mathscr{M}^{1}(\mathbb{A})$, and let $\eta$ denote a nonnegative measure on $\mathbb{U} \backslash\{e\}$ such that $\eta$ is a bounded measure outside any neighbourhood of the unit.

(b) $\mu$, respectively, $\eta$ possess finite first-order moments if $\int_{\mathbb{H}} f \mathrm{~d} \mu<\infty$, respectively, $\int_{\mathbb{H} \backslash U} f \mathrm{~d} \eta<\infty$ for all subadditive
Borel functions $f: \mathbb{W} \rightarrow \mathbb{R}_{+}$and $U=U_{f, \epsilon} \in \mathfrak{U}_{\mathbb{}}$ are defined previously.

(c) Analogously, we define for a group $\mathbb{\square}=N=$ $C_{K}(\tau): \mu$, respectively, $\eta$ possess finite logarithmic moments if $\int_{N} \log (1+f) \mathrm{d} \mu<\infty$, respectively, $\int_{\mathbb{⿴ 囗 U} U} \log (1+f) \mathrm{d} \eta<\infty$ for all regular $\tau$-functions $f, U=U_{f, \epsilon} \in \mathfrak{U}_{\llbracket}$ as in (c).

In fact, if the integrals $\int_{\mathbb{W} U} f \mathrm{~d} \eta$ in (b), respectively, $\int_{\mathbb{M l U}} \log (1+f) \mathrm{d} \eta$ in (c) are finite for some $U=U_{f, \epsilon}$ (as above), they are finite for all $U \in \mathfrak{U}_{\sharp}$. And if the integrals are finite for some relatively compact $U_{0} \in \mathcal{U}_{\sharp}$, they are finite for all $U \in \mathfrak{U}_{\mathbb{H}}$.

Remark 2. The definition of logarithmic moments depends on $\tau$-functions and hence on $K$ and on the particular automorphism $\tau$. If $\mathbb{U}=\mathbb{G}=N \rtimes_{\tau} \mathbb{Z}$ and $f$ is a regular $\tau$ function, and if we define $U=\{f \leq 1\}, V:=U \otimes\{1\} \cup U \otimes$ $\{0\} \cup\{U \otimes\{1\}\}^{-1}$, then $\mathbb{G}$ is generated by $V$ and $\log (1+f) \sim$ $\delta_{V}(\cdot, 0)$ (cf. [1, Proposition 2.14].) If $W$ is an other relatively compact symmetric neighbourhood generating $\mathbb{G}$, then $\delta_{V} \sim$ $\delta_{W}$ (see [28, Remarque 3].) Hence $\lambda \in \mathscr{M}^{1}(\mathbb{G})$ possesses finite first-order moments if $\delta_{V}$, equivalently, $\delta_{W}$ is integrable. Hence $\lambda=\mu \otimes \varepsilon_{k}$ possesses finite first-order moments iff $\left.\int_{N} \delta_{V}\right|_{N} \mathrm{~d} \mu<\infty$ (see [1, Proposition 2.14, Corollary 2.15].)

Note that for contractible groups logarithmic moments are frequently defined in terms of "group-norms" or "homogeneous norms" (e.g., in [29, 2.6, 2.11], [6, 2.7.26 (f)]), [30]), generalizing the situation of vector spaces. We show in Lemma A.2 and Theorem 22 (cf. Remark 21) that on homogeneous groups this definition is equivalent to Definition 1 . In fact, if $\tau$ is a contracting automorphism, then there exist $k \in N$ and a continuous group $T=\left(\tau_{t}\right)$ such that $\tau^{k} \in T$ (cf., e.g., [6, 2.8.14]), and therefore, Lemma A.2, respectively, Theorem 22 apply also for discrete times.

Fact 2. Let $N$ be a locally compact group; $\tau \in \operatorname{Aut}(N)$, $N=C_{K}(\tau)$, and, as before, $\mathbb{G}=N \rtimes_{\tau} \mathbb{Z}$. Let $\nu(k)=$ $\star_{j=0}^{k-1} \tau^{j}(\nu(1)) .\left(\omega_{K}\right.$ denotes the normalized Haar measure on the compact subgroup $K \subseteq N$.) Then the following assertions are equivalent:

(i) $v(k)$ is weakly convergent $\bmod K$; that is, $\lim _{k} v(k) \star$ $\omega_{K}$ exists.

(ii) $v(1)$ possesses finite logarithmic moments.

(iii) $\lambda=\nu \otimes \varepsilon_{1}$ possesses finite first-order moments.

【The equivalence of the assertions "(i)-(ii)" is shown in the context of invariant metrics on $N / K$, respectively, of regular $\tau$-functions, in [1, Theorem 3.9, Propositions 4.2 and 4.3]. For "(ii) $\Leftrightarrow$ (iii)" see [1, Corollary 2.15].]

The equivalence of the conditions "(i)-(ii)" (as well as equivalence with stochastic and almost sure convergence of corresponding random variables) is folklore for vector spaces (cf. [31], or e.g., the monograph [32, Lemma 3.6.5]), for homogeneous groups [29], [6, 2.14.24], for general contractible groups [30] (in the context of "group norms".) As long as almost sure convergence of corresponding random variables is considered, Lévy's equivalence theorem is involved, and 
hence the underlying group is usually supposed to be second countable. In the situation here a superfluous condition as $N / K$ is metrizable, cf. [1, Theorems 2.5 , Proof of Theorem 3.9]. Furthermore, in most cases, metrizability may be supposed without loss of generality.

Remark 3. The groups $\mathbb{G}$ and $N$, generated by the supports of the random walk, respectively, the M-semigroup (cf. Definition 4 below) are $\sigma$-compact. Therefore, $\mathbb{G}$ and $N$ are representable as projective limits of second countable locally compact groups (cf. [33, page 101, Example 11] or [34, Theorem 5.2]): $N=\lim _{\leftarrow} N / \Gamma_{\alpha}$, respectively, $\mathbb{G}=$ $\lim _{\leftarrow}\left(N / \Gamma_{\alpha}\right) \rtimes_{\tau_{\alpha}} \mathbb{Z}$, where $\Gamma_{\alpha}$ are compact, $\tau$-invariant subgroups $\Gamma_{\alpha} \triangleleft N$ and $\tau_{\alpha} \in \operatorname{Aut}\left(N / \Gamma_{\alpha}\right)$. Therefore in many investigations second countability may be assumed w.l.o.g. For example, $\nu(k) \star \omega_{K}$ is convergent, respectively, $\{\nu(k)\}$ is relatively compact, respectively, satisfies (2) below iff for all $\alpha$ the projections to the quotients share this property.

Definition 4. (a) A sequence $\{\nu(k)\}_{k \in \mathbb{Z}_{+}}$in $\mathscr{M}^{1}(N)$ satisfying

$$
\begin{gathered}
\nu(0)=\varepsilon_{e}, \quad \nu(1)=: \nu, \\
\nu(k+\ell)=\nu(k) \star \tau^{k}(\nu(\ell)), \quad k, \ell \in \mathbb{Z}_{+}
\end{gathered}
$$

is called discrete M-(convolution)-semigroup (also called Mehler semigroup, $\tau$-semigroup, skew convolution semigroup, etc.)

Note that we are mainly interested in the behaviour of the random walk, respectively, the $\mathrm{M}$-semigroup for large times $k$; hence the definition of $\lambda^{0}=\nu(0) \otimes \varepsilon_{0}$ is of minor importance. We could replace $\lambda^{0}$ by an idempotent $\omega_{H} \otimes \varepsilon_{e}$ such that $\lambda^{0} * \lambda * \lambda^{0}=\lambda$, that is, $\omega_{H} \star \nu \star \tau\left(\omega_{H}\right)=\nu$. $\omega_{H}$ denoting the Haar measure on a compact subgroup $H \subseteq N$.

(b) $\rho \in \mathscr{M}^{1}(N)$ is called $\tau$-decomposable, if for some cofactor $v=v(1) \in \mathscr{M}^{1}(N)$ we have $\rho=v \star \tau(\rho)$. Then by induction, $\rho=\nu(k) \star \tau^{k}(\rho)$. $\operatorname{Cof}_{\rho}\left(\tau^{k}\right)$ denotes the set of cofactors of $\rho$ with respect to $\tau^{k}$. As immediately seen, one can choose $\nu(k)=\star_{j=0}^{k-1} \tau^{j}(\nu(1))$ for $k \geq 2$; hence the cofactors can be chosen as (discrete) M-semigroup $\{\nu(k)\}$.

(c) $\rho$ is called right $K$-invariant if $\rho \star \omega_{K}=\rho$ (for some compact subgroup $K$ ).

(d) For short, $\rho$ is $K$ - $\tau$-decomposable if $\rho$ is $\tau$ decomposable and right $K$-invariant.

$\operatorname{Cof}_{\rho}(\tau)$ is closed, hence according to the shiftcompactness theorem (cf. [35, III, Theorems 2.1 and 2.2] (for metrizable groups), [36, Theorem 1.2.21]), $\operatorname{Cof}_{\rho}(\tau)$ is compact in $\mathscr{M}^{1}(N)$. If $\left\{\tau^{k}(\rho)\right\}$ is relatively compact, in particular, if $N=C_{K}(\tau)$, then, again by the shift-compactness theorem, $\bigcup_{k \geq 0} \operatorname{Cof}_{\rho}\left(\tau^{k}\right)$ is relatively compact.

Remark 5. In the following, we distinguish carefully between adapted and nonadapted space-time random walks. Let $\left\{\lambda^{k}=\right.$ $\left.\nu(k) \otimes \varepsilon_{k}\right\}$ be a nondissipating space-time random walk on $\mathbb{G}=N \rtimes_{\tau} \mathbb{Z}$ with relatively compact M-semigroup $\{\nu(k)\} \subseteq$ $\mathscr{M}^{1}(N)$. If the random walk $\left\{\lambda^{k}\right\}$ is not adapted let $\mathbb{G}_{\lambda} \subseteq$ $\mathbb{G}$ denote the closed subgroup generated by supp $\lambda$. Hence, on $\mathbb{G}_{\lambda},\left\{\lambda^{k}\right\}$ is nondissipating and adapted. Therefore there exists a closed subgroup $N_{\lambda}$ in $\mathbb{G}$, normal in $\mathbb{G}_{\lambda}$, containing the supports supp $\lambda^{k} * \tilde{\lambda}^{k}$, and $y \in \mathbb{G}_{\lambda}$, such that with $\tau^{\prime}:=\left.i_{y}\right|_{N_{\lambda}} \in \operatorname{Aut}\left(N_{\lambda}\right)$ there exists a relatively compact M-semigroup (with respect to $\left.\tau^{\prime}\right)\left\{\nu^{\prime}(k)\right\} \subseteq \mathscr{M}^{1}\left(N_{\lambda}\right)$, such that $\lambda^{k}=\nu^{\prime}(k) * \varepsilon_{y^{k}}$ for $k \in \mathbb{Z}_{+}$. Let $y=(z, 1)$; hence $y^{k}=(z(k), k)$ with $z=z(1), z(k) \in N$. Then $y N_{\lambda}=N_{\lambda} y$ iff $\tau\left(N_{\lambda}\right)=i_{z^{-1}}\left(N_{\lambda}\right)=z^{-1} N_{\lambda} z$. Note that $\tau^{\prime}=i_{z} \circ \tau$ since $y=(z, 0)(e, 1)$.

Furthermore, $\lambda=\nu \otimes \varepsilon_{1}=\nu * \varepsilon_{(e, 1)}, \nu=\nu(1)$, and $\lambda=$ $\nu^{\prime} * \varepsilon_{(z, 1)}=\left\{\nu^{\prime} \star \varepsilon_{z}\right\} \otimes \varepsilon_{1}$. Hence $\nu=\nu^{\prime} \star \varepsilon_{z}$, analogously, $\nu(k)=\nu^{\prime}(k) \star \varepsilon_{z(k)}, k \in \mathbb{Z}_{+}$. And thus supp $\nu(k) \subseteq N_{\lambda} \cdot z(k)$.

$N_{\lambda}$ is the smallest closed normal subgroup of $\mathbb{G}_{\lambda}$ containing supp $\lambda^{k} * \widetilde{\lambda}^{k}$, and $\lambda^{k} * \widetilde{\lambda}^{k}=v(k) \star \widetilde{\nu(k)}=\nu^{\prime}(k) \star \widetilde{\nu^{\prime}(k)}$; hence it follows that a nondissipating space-time random walk $\left\{\lambda^{k}=\nu(k) \otimes \varepsilon_{k}\right\}$ is adapted on $\mathbb{G}$ iff for any closed subgroup $N^{\prime} \subseteq N$ and $z \in N$, with $(z, 1)$ normalizing $N^{\prime}$ and satisfying

$$
\begin{aligned}
& \text { (1) } N^{\prime} \supseteq \operatorname{supp} v(1) \star \widetilde{v(1)}, \\
& \text { (2) } \tau\left(N^{\prime}\right)=z^{-1} N^{\prime} z \Longrightarrow N^{\prime}=N .
\end{aligned}
$$

In this sense we may w.l.o.g. assume that a nondissipating random walk $\left\{\lambda^{k}=\nu(k) \otimes \varepsilon_{k}\right\}$ on a locally compact group $\mathbb{G}$ is adapted, respectively, a relatively compact $M$ semigroup $\{\nu(k)\}$ on a locally compact group $N$ (with respect to $\tau \in \operatorname{Aut}(N)$ ) satisfies condition (3). (If necessary replacing $\mathbb{G}, N, \tau, \nu(k)$ by $\mathbb{G}_{\lambda}, N^{\prime}, \tau^{\prime}=i_{z} \circ \tau, \nu^{\prime}(k)=\nu(k) \star \varepsilon_{z(k)}$ as previously mentioned.)

Relations between nondissipative random walks, Msemigroups, and $\tau$-decomposable laws are collected in the following.

Theorem 6. (a) Let $\mathbb{G}$ be a locally compact group and $\left\{\lambda^{k}\right\} \subseteq$ $\mathscr{M}^{1}(\mathbb{G})$ a nondissipating adapted random walk. Then there exist a closed normal subgroup $N$ and $x \in \mathbb{G}$, such that with $\tau:=\left.i_{x}\right|_{N} \in \operatorname{Aut}(N)$, one has $\mathbb{G} \cong N \rtimes_{\tau} \mathbb{Z}, N=C_{K}(\tau)$ for a compact, $\tau$-invariant subgroup $K \subseteq N$. Furthermore, the random walk is representable as $\left\{\lambda^{k}=\nu(k) \otimes \varepsilon_{k}\right\}$, where $\{\nu(k)\} \subseteq \mathscr{M}^{1}(N)$ is a relatively compact M-semigroup (with respect to $\tau$ ), and condition (3) is satisfied.

(b) Conversely, let $N$ be a locally compact group, $\tau \in$ $\operatorname{Aut}(N)$, and let $\{\nu(k)\}$ be a relatively compact $M$-semigroup in $M^{1}(N)$, and assume condition (3) to be satisfied. Then the space-time random walk $\left\{\lambda^{k}=\nu(k) \otimes \varepsilon_{k}\right\} \subseteq \mathscr{M}^{1}(\mathbb{G})$ is nondissipating and adapted. ( $\mathbb{G}$ denotes $N \rtimes_{\tau} \mathbb{Z}$ ). Hence (a) applies. In particular, $N=C_{K}(\tau)$ for some compact $\tau$-invariant subgroup $K \subseteq N$.

(c) Non-dissipative random walks, respectively, relatively compact $M$-semigroups define $\tau$-decomposable laws.

In fact, in case (a) or (b) we obtains that $\rho:=\lim _{k} \nu(k)$ * $\omega_{K} \in \mathscr{M}^{1}(N)$ exists and is $K$ - $\tau$-decomposable.

(d) Conversely, $\tau$-decomposable laws define nondissipating random walks, respectively, relatively compact $M$-semigroups.

Precisely, assume that $N=C_{K}(\tau)$. Let $\rho \in \mathscr{M}^{1}(N)$ be $K$ - $\tau$-decomposable. Then there exists a M-semigroup $\{\nu(k)\}$ of 
cofactors of $\rho$ such that $\nu(k) \star \omega_{K} \rightarrow \rho$. In particular, $\{\nu(k)\}$ is relatively compact, and then and hence (a), respectively, (b) apply.

(e) Let $\mathbb{G}=N \rtimes_{\tau} \mathbb{Z}, N=C_{K}(\tau)$. An adapted space-time random walk $\left\{\lambda^{k}=\nu(k) \otimes \varepsilon_{k}\right\} \subseteq \mathscr{M}^{1}(\mathbb{G})$ is nondissipating, respectively, a $M$-semigroup $\{\nu(k)\} \subseteq \mathscr{M}^{1}(N)$ satisfying (3) is relatively compact iff $\nu=v(1)$ possesses finite logarithmic moments, respectively, iff $\lambda$ possesses finite first-order moments.

Proof. For (a), (b) see Fact 1 and the discussion in Remark 5.

(c) Let $\{\nu(k)\}$ be a relatively compact M-semigroup on $N=C_{K}(\tau)$. Since $\left(\tau^{k}\right)$ is uniformly $K$-contracting on compact subsets, the accumulation points of $\left\{\tau^{k}(\nu(n))\right\}_{n \in \mathbb{Z}_{+}, k \rightarrow \infty}$ are supported by $K$. Assume, for some subnets, that $\nu\left(k_{n}\right) \rightarrow$ $\alpha$ and $\nu\left(\ell_{n}\right) \rightarrow \beta$ and, w.l.o.g., $k_{n} \leq \ell_{n}$ for all $n$. Then $\nu\left(\ell_{n}\right) \star \omega_{K} \rightarrow \beta \star \omega_{K}$ on the one hand, $\nu\left(\ell_{n}\right) \star \omega_{K}=\nu\left(k_{n}\right) \star$ $\tau^{k_{n}}\left(\nu\left(\ell_{n}-k_{n}\right)\right) \star \omega_{K}$ with $\lim \tau^{k_{n}}\left(\nu\left(\ell_{n}-k_{n}\right)\right) \star \omega_{K}=\omega_{K}$ on the other. Hence $\alpha \star \omega_{K}=\beta \star \omega_{K}$ follows. That is, $\rho=\lim \nu(k) \star \omega_{K}$ exists and is obviously right $K$-invariant.

Furthermore, $\rho=\lim \nu(k+1) \star \omega_{K}=\lim \nu(1) \star \tau(\nu(k) \star$ $\left.\omega_{K}\right)=\nu(1) \star \tau(\rho)$ yields $K$ - $\tau$-decomposability of $\rho$.

(d) Conversely, assume $\rho$ to be $K$ - $\tau$-decomposable. $\left\{\tau^{k}(\rho)\right\}$ is relatively compact as $\tau$ is $K$-contracting, and all accumulation points are supported by $K$. Right $K$-invariance of $\rho$ implies that $\tau^{k}(\rho) \rightarrow \omega_{K}$. As afore mentioned, we can choose $\nu(k)=\star_{j=0}^{k-1} \tau^{j}(\nu), \nu=\nu(1)$ in $\operatorname{Cof}_{\rho}(\tau)$, hence as an M-semigroup of cofactors. Hence $\rho=\nu(k) \star \tau^{k}(\rho)$ and $\tau^{k}(\rho) \rightarrow \omega_{K}$ yield (1) relative compactness of $\{\nu(k)\}$ and (2) $\nu(k) \star \omega_{K} \rightarrow \rho$ according to the shift-compactness theorem.

(e) Follows by Fact 2, Remark 3, and [1, Corollary 2.15, resp., Proposition 4.3].

More generally we have the following.

Remark 7. (a) Let $N$ be a locally compact group, $\tau \in \operatorname{Aut}(N)$, $\mathbb{G}=N \rtimes_{\tau} \mathbb{Z}$ as previously mentioned. Let $\{\nu(k)\},\left\{\lambda^{k}=\right.$ $\left.\nu(k) \otimes \varepsilon_{k}\right\}$ be a M-semigroup, respectively, the corresponding space-time random walk. As immediately seen, if the Msemigroup is only supposed to be relatively shift compact, then the random walk is nondissipating. And if the random walk is not adapted, Remark 5 applies.

(b) Let $N$ be a locally compact group, $\tau \in \operatorname{Aut}(N)$, and let $\rho \in \mathscr{M}^{1}(N)$ be $\tau$-decomposable, with $\{\nu(k)\}$ as M-semigroup of cofactors. Then $\rho=\nu(k) \star \tau^{k}(\rho), k \in \mathbb{Z}_{+}$, yields that $\{\nu(k)\}$ is relatively shift compact and therefore (a) applies.

(c) If in (b) in addition $\left\{\tau^{k}(\rho)\right\}$ is assumed to be relatively compact (e.g., if $N=C_{K}(\tau)$ as in Theorem 6), then, again by the shift-compactness theorem, the M-semigroup of cofactors is already relatively compact.

Note that in Theorem 6 and Remark 7, if $N=C_{K}(\tau)$ and $K \neq\{e\}, \lim _{k} \nu(k)$ will not in general exist. See for example, Example 3.16 in [1], with compact $N=\mathbb{T}^{2}$ and an infinite number of accumulation points. Further types of examples are obtained in the following way.
Example 8. (a) Let $\Gamma$ be a contractible locally compact group, with contracting $\sigma \in \operatorname{Aut}(\Gamma)$, hence $\Gamma=C(\sigma)$. Let $\{\mu(k)\} \subseteq$ $\mathscr{M}^{1}(\Gamma)$ be a relatively compact M-semigroup, $\mu(k+\ell)=\mu(k)$ * $\sigma^{k}(\mu(\ell))$. As $\sigma^{k}\{\mu(\ell): \ell \geq 0\} \stackrel{k \rightarrow \infty}{\longrightarrow}\left\{\varepsilon_{e}\right\}, \lim \mu(k)=: \rho_{1}$ exists (Theorem 6(c) with $K=\{e\}$ ). Let $H$ be a finite cyclic group, let $x_{0} \in H$ generating $H$ with $\operatorname{ord}\left(x_{0}\right)>2$, and assume for some $\xi \in \operatorname{Aut}(H)$ that $\xi\left(x_{0}\right)=x_{0}^{-1}$ (e.g., $\xi: x \mapsto x^{-1}$ ). Put $N:=\Gamma \otimes H$, define $\tau \in \operatorname{Aut}(N)$ as $\tau=\sigma \otimes \xi$, and put finally $\nu=\nu(1):=\mu(1) \otimes \varepsilon_{x_{0}}$. Then $\left\{\nu(k)=\mu(k) \otimes \varepsilon_{y(k)}\right\}$ is a relatively compact M-semigroup (with respect to $\tau$ ) in $\mathscr{M}^{1}(N)$, where $y(k)=\prod_{j=0}^{k-1} \xi^{j}\left(x_{0}\right)$. But $y(k)=e$ and $y(k)=$ $x_{0}$, both infinitely often; hence $\nu(k)$ is not convergent. In fact, $\operatorname{LIM}\{\nu(k)\}=\left\{\rho_{1} \otimes \varepsilon_{e}, \rho_{1} \otimes \varepsilon_{x_{0}}\right\}$. But we have $\nu(k) \star \omega_{H} \rightarrow$ $\rho_{1} \otimes \omega_{H}$.

(b) A trivial case: let $K(=N)$ be a monothetic compact group with dense subgroup $\left\{x(k):=x_{0}^{k}: k \in \mathbb{Z}\right\} \cong \mathbb{Z}$ (for some $\left.x_{0} \in K\right)$. Put $\tau:=i d$, obviously $K$-contracting; hence $\mathbb{G}=K \otimes \mathbb{Z}$. Then $\left\{\nu(k):=\varepsilon_{x(k)}\right\}_{k \in \mathbb{Z}_{+}}$is a M-semigroup (with respect to $\tau$ ), which is relatively compact in $\mathscr{M}^{1}(K)$. But $\nu(k)$ is not convergent, in fact, $\operatorname{LIM}\{\nu(k)\}=\left\{\varepsilon_{\kappa}: \kappa \in K\right\}$. Here we have $\nu(k) \star \omega_{K} \equiv \omega_{K}$.

In [12, Theorem 1], it is shown for a nondissipating random walk $\left\{\lambda^{k}=\nu(k) \otimes \varepsilon_{k}\right\}$ that there exists a measurable dense $\tau$-invariant subgroup $N_{1} \subseteq N$ on which a sequence $\tau^{q_{n}}$ acts as contracting mod. a compact subgroup $H$, and $\lambda^{n}=\left(\nu \otimes \varepsilon_{1}\right)^{n}$ are concentrated on cosets of $N_{1}$. Hence $\tau$ is weakly contracting mod. $H$ on $N$ (cf. the definition in [12]), and thus $N=C_{K}(\tau)$, for some $\tau$-invariant subgroup $K \supseteq H$ [12, Theorem 5]. In interesting examples, for example, in the context of (semi-) stable laws, we have $N_{1}=C_{H}(\tau)$ for a compact $\tau$-invariant subgroup $H \subseteq K$. In fact, investigations of the structure of contractible and $K$-contractible subgroups had also been pushed forward in connection with investigations of (semi-) stable laws. See for example, [4$6,10]$ and the literature mentioned there. However, concentration functions were not used as an essential tool (except in connection with random time substitutions and geometric (semi-) stability, cf. [6, 37]). Nevertheless it is worth to point out that semistable laws provide interesting examples of relatively compact $\mathrm{M}$-semigroups, hence of nondissipating random walks.

Example 9 (cf., e.g., $[6, \S 3.5])$. Let $\left\{\rho_{t}\right\}_{t \geq 0}$ be a continuous convolution semigroup in $\mathscr{M}^{1}(N)$, and let $N$ be a locally compact group. Let $\tau \in \operatorname{Aut}(N)$ and $0<c<1 .\left\{\rho_{t}\right\}$ is (strictly) $(\tau, c)$-semistable if for all $t \geq 0, \tau\left(\rho_{t}\right)=\rho_{t \cdot c}$. The idempotent $\rho_{0}=\omega_{H}$ is a normalized Haar measure on a compact $\tau$-invariant subgroup $H$. If $N$ is second countable, the contraction subgroups $C(\tau)$ and $C_{H}(\tau)$ are Borel sets, and we have $\rho_{t}\left(C_{H}(\tau)\right)=1$ for all $t$. Hence we assume that $N=\left(C_{H}(\tau)\right)^{-}$, and therefore $N=C_{K}(\tau)$ (as mentioned previously).

Let now $\rho:=\rho_{1}$. Then $\rho=\rho_{1-c^{n}} \star \rho_{c^{n}}=\rho_{1-c^{n}} \star \tau^{n}(\rho)$. Hence $\rho$ is $H$ - $\tau$-decomposable with cofactors $\nu(n):=\rho_{1-c^{n}} \in$ $\operatorname{Cof}_{\rho}\left(\tau^{n}\right)$. In particular, $\rho_{1-c^{n}}$ (and hence all $\rho_{t}$ ) possess finite logarithmic moments. (This could also be proved by direct 
calculation.) In that example, $\nu(n)$ are right $H$-invariant and $\lim \nu(n)=\rho$ exists. (And thus trivially also $\lim \nu(n) \star \omega_{K}=$ $\left.\rho \star \omega_{K}\right)$.

If $C(\tau)$ is closed and $C_{H}(\tau)=C(\tau) \cdot H$, then $C_{H}(\tau)$ is closed, hence $H=K$ and $N=C_{K}(\tau) . C(\tau)$ is known to be closed if there exist contracting continuous one-parameter groups of automorphisms [5] moreover for $p$-adic Lie groups [38] and more generally for totally disconnected groups if $\tau$ is a "tidy" automorphism [14]. And we have $C_{H}(\tau)=C(\tau) \cdot H$, at least in the case of totally disconnected groups or of Lie groups. See [11]; see also [13-15] for previous results. For Lie groups see [5], [6, Theorem 3.2.13]. For more information concerning the decomposition property $C_{H}(\tau)=C(\tau) \cdot H$, cf. $[39,40]$. However, $C(\tau)$ will not in general be closed, not even on a 2-dimensional torus (cf., e.g., [5], [6, Example 3.12.5]). Then, as mentioned previously if w.l.o.g. $N=\left(C_{H}(\tau)\right)^{-}, \tau$ is weakly contracting $\bmod H$ on $N$, and hence $N=C_{K}(\tau)$ for some compact, $\tau$-invariant subgroup $K \supseteq H$. Note that, if $C_{H}(\tau)$ is not closed, additional conditions are needed to guarantee existence of $\lim _{k} \nu(k) \star \omega_{H}$ (cf. Example 8 above).

The following result will explain in more detail the interplay between limit behaviour of relatively compact Msemigroups and $\tau$-decomposability.

Proposition 10. Let $N=C_{K}(\tau)$. Let $\{\nu(k)\}$ be a relatively compact M-semigroup. To avoid measurability problems, $N$ is supposed to be second countable. Put $\mathscr{A}:=\operatorname{LIM}\{\nu(k): k \rightarrow$ $\infty\}$ and $\mathcal{S}:=\operatorname{LIM}\left\{\tau^{n}\left(\nu\left(k_{n}\right)\right): n \rightarrow \infty,\left(k_{n}\right) \subseteq \mathbb{Z}_{+}\right\} \subseteq$ $\mathscr{M}^{1}(K)$.

Then one has the following

(a) $\mathscr{A} \subseteq \rho \star \mathcal{S}($ for any $\rho \in \mathscr{A})$.

(b) $\tau(\mathcal{S})=\mathcal{S}$.

(c) If at least some $\rho_{0} \in \mathscr{A}$ and $\mathcal{S}$ belong to a commutative $\star$-sub-semigroup $\mathscr{M}$ of $\mathscr{M}^{1}(N)$, then any $\rho \in$ $\mathscr{A}$ is $\tau$-decomposable with $v(k) \star \alpha_{k} \in \operatorname{Cof}_{\rho}\left(\tau^{k}\right)$ for some $\alpha_{k} \in \mathcal{S}, k \in \mathbb{Z}_{+}$.

(d) If $\nu(k)\left(C_{H}(\tau)\right)=1$ for some compact $\tau$-invariant subgroup $H \subseteq K$ and $\nu(k) \star \omega_{H}=\nu(k)$, for all $k \geq 1$, and if one assumes in addition that $\mathcal{S} \subseteq \mathscr{M}^{1}(H)$, then $\rho=\lim _{k} \nu(k)$ exists, and $\rho$ is $H$ - $\tau$-decomposable with cofactors $v(k) \in \operatorname{Cof}_{\rho}\left(\tau^{k}\right)$.

Proof. By assumption, $\left(\tau^{k}\right)$ is compactly contracting $\bmod K$ on $N$.

(a) Let $\rho, \sigma \in \mathscr{A}, \nu\left(k_{n}\right) \rightarrow \rho$ and $\nu\left(\ell_{n}\right) \rightarrow \sigma$. Assume that that w.l.o.g. $\ell_{n} \geq k_{n}$ for all $n$ (else pass to subsequences). Then $\nu\left(\ell_{n}\right)=\nu\left(k_{n}\right) \star \tau^{k_{n}}\left(\nu\left(\ell_{n}-k_{n}\right)\right) \rightarrow$ $\rho \star \alpha, \alpha \in \mathcal{S}$. Hence $\sigma=\rho \star \alpha \in \rho \star \mathcal{S}$, and analogously, $\rho \in \sigma \star \mathcal{S}$ follows.

(b) Assume that $\tau^{\ell_{n}}\left(\nu\left(m_{n}\right)\right) \rightarrow \gamma \in \mathcal{S}$. Then $\tau(\gamma)=$ $\lim \tau^{\ell_{n}+1}\left(\nu\left(m_{n}\right)\right)$; hence $\tau(\gamma) \in \mathcal{S}$, and analogously $\tau^{-1}(\gamma) \in \mathcal{S}$ follows.

(c) Let $\rho \in \mathscr{A}$; hence $\rho=\rho_{0} \star \alpha$ for some $\alpha \in \mathcal{S}$. Let $\nu\left(k_{n}\right) \rightarrow \rho$. w.l.o.g. assume that $\rho=\rho_{0}$, else replace in the following $\rho$ by $\rho_{0} \star \alpha$. Then, for all $j \in \mathbb{N}, \nu\left(k_{n}\right)=$ $\nu(j) \star \tau^{j}\left(\nu\left(k_{n}-j\right)\right)$ and $\nu\left(k_{n}-j\right) \rightarrow \rho \star \beta_{j}$ (along a subsequence) for some $\beta_{j} \in \mathcal{S}$. Hence $\nu\left(k_{n}\right) \rightarrow \nu(j) \star$ $\tau^{j}(\rho) \star \tau^{j}\left(\beta_{j}\right)=\left(\nu(j) \star \tau^{j}\left(\beta_{j}\right)\right) \star \tau^{j}(\rho)$. That is, $\nu(j) \star$ $\tau^{j}\left(\beta_{j}\right) \in \operatorname{Cof}_{\rho}\left(\tau^{j}\right)$. Whence the assertion with $\alpha_{j}=$ $\tau^{j}\left(\beta_{j}\right)$.

(d) Since by assumption $\nu(k)$ are right $H$-invariant, the accumulation points $\alpha \in \mathcal{S}$ are right $H$-invariant and supported by $H$; thus $\mathcal{S}=\left\{\omega_{H}\right\}$. Whence $\mathscr{A}=\{\rho\}$ and thus $v(k) \rightarrow \rho$.

The following example will illustrate Proposition 10.

Example 11. Let $N=C_{K}(\tau)$ as before.

(a) Let $\left\{\rho_{t}\right\} \subseteq \mathscr{M}^{1}(N)$ be a $(\tau, \alpha)$-semistable continuous convolution semigroups (as in Example 9), and let $\xi \in$ $\mathscr{M}^{1}\left(\mathbb{R}_{+}\right)$be $H_{\alpha}$-decomposable with cofactor $\eta$, with $H_{\alpha}$ denoting the homothetic transformation $x \mapsto \alpha \cdot x$ on $\mathbb{R}$. Then the subordinated measure $\lambda:=\int_{\mathbb{R}_{+}} \rho_{t} \mathrm{~d} \xi(t)$ is $\tau$-decomposable with cofactor $\int_{\mathbb{R}_{+}} \rho_{t} \mathrm{~d} \eta(t)$. The set $\mathscr{M}:=\left\{\int_{\mathbb{R}_{+}} \rho_{t} \mathrm{~d} \gamma(t)\right.$ : $\left.\gamma \in \mathscr{M}^{1}\left(\mathbb{R}_{+}\right)\right\}$is a commutative sub-semigroup of $\mathscr{M}^{1}(N)$ containing $\lambda$ and the cofactors (cf. Proposition 10(c)).

Let $N^{\prime}:=C_{H}(\tau)$ for some compact $\tau$-invariant subgroup $H \subseteq K$. Assume $\rho_{t}\left(N^{\prime}\right)=1$ for all $t$, then also $\lambda\left(N^{\prime}\right)=1$ and $v\left(N^{\prime}\right)=1$ for all $v \in \mathscr{M}$.

(b) Let $\rho^{(i)} \in \mathscr{M}^{1}(N)$ be $\tau$-decomposable with cofactors $v^{(i)}(k), i=1,2$. [e.g., let $\left\{\rho_{t}^{(i)}\right\}$ be $\left(\tau, \alpha_{i}\right)$-semistable continuous convolution semigroups and $\rho^{(i)}=\rho_{1}^{(i)}$. \Assume that $\nu^{(2)}(1)$ and $\tau\left(\rho^{(1)}\right)$ commute. 【e.g., for semistable laws, assume that $\rho_{t}^{(1)}, \rho_{s}^{(2)}$ commute for all $s$, t. Then $\rho:=\rho^{(1)} \star \rho^{(2)}$ is $\tau$ decomposable with cofactor $\nu^{(1)}(1) \star \nu^{(2)}(1)$. «But note that if $\rho^{(i)}$ are semistable and if $\alpha_{1} \neq \alpha_{2}-\rho_{1}^{(1)} \star \rho_{1}^{(2)}$ is in general $\operatorname{not}(\tau, \beta)$-semistable for $0<\beta<1$.

If $\rho^{(i)}\left(C_{H_{i}}(\tau)\right)=1$, for $\tau$-invariant compact subgroups $H_{i} \subseteq K$, then $\rho\left(C_{H_{1}}(\tau) \cdot C_{H_{2}}(\tau)\right)=1$. If $\omega_{H_{1}}$ and $\omega_{H_{2}}$ commute, then $H_{1} \cdot H_{2}=: H_{3}$ is a compact $\tau$-invariant subgroup and $C_{H_{3}}(\tau) \supseteq\left\langle C_{H_{1}}(\tau) \cdot C_{H_{2}}(\tau)\right\rangle$, the subgroup generated by $C_{H_{i}}(\tau), i=1,2$.

(c) In general, without commutativity assumptions, $\rho$ in (b) will not in general be $\tau$-decomposable. In particular, for point measures $\varepsilon_{x}, x \in N$ we obtain: $\bar{\rho}=\rho^{(1)} \star \varepsilon_{x}$ is in general not $\tau$-decomposable. In fact, without commutativity assumption, the left shifted measure, $\rho:=\varepsilon_{x} \star \rho^{(1)}$, is, as easily seen, $\tau$-decomposable with cofactors $\nu(k)=\varepsilon_{x} \star v^{(1)}(k) \star$ $\varepsilon_{\tau^{k}\left(x^{-1}\right)}$. Representing $\bar{\rho}$ as $\varepsilon_{x} \star i_{x^{-1}}\left(\rho^{(1)}\right)$ we see that $\bar{\rho}$ is $\bar{\tau}$ decomposable, with $\bar{\tau}:=i_{x^{-1}} \circ \tau \circ i_{x}$, but in general not $\tau$ decomposable.

(d) To simplify notations we assume that $H_{i}=\{e\}$. Let $N^{\prime}:=C(\tau)$ and assume that now $\rho^{(1)}\left(N^{\prime}\right)=v^{(1)}(k)\left(N^{\prime}\right)=$ 1. Then $\rho$ and $\nu(k)$ (in (c)) are concentrated on cosets of compactly contractible subgroups of $N: \rho\left(x N^{\prime}\right)=1$ and $\nu(k)\left(x N^{\prime} \tau^{k}\left(x^{-1}\right)\right)=1$. Hence, with $N^{\prime \prime}:=i_{x}\left(N^{\prime}\right)$ we have: $\rho\left(N^{\prime \prime} x\right)=1$ and $\nu(k)\left(N^{\prime \prime} x \tau^{k}\left(x^{-1}\right)\right)=1$. Furthermore, $N^{\prime \prime}=$ $C_{\bar{H}}(\bar{\tau})$, where $\bar{\tau}:=i_{x} \circ \tau \circ i_{x^{-1}}, \bar{H}:=i_{x}(H)$. 
(e) In case (c), (d) we determine the sets of accumulation points $\mathcal{S}$ and $\mathscr{A}$ (Proposition 10):

$\mathcal{S}=\operatorname{LIM}\left\{\tau^{n}\left(\nu\left(k_{n}\right)\right)\right\}=\operatorname{LIM}\left\{\varepsilon_{\tau^{n}(x)} \star \tau^{n}\left(\nu^{(1)}\left(k_{n}\right)\right) \star \varepsilon_{\tau^{n+k_{n}}\left(x^{-1}\right)}\right\}$,

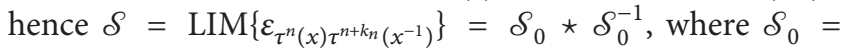
$\operatorname{LIM}\left\{\varepsilon_{\tau^{n}(x)}\right\}$. 【In fact, obviously $\mathcal{S} \subseteq \mathcal{S}_{0} \star \mathcal{S}_{0}^{-1}$. On the other hand, assume $\tau^{k(n)}(x) \rightarrow z$ and $\tau^{\ell(n)}(x) \rightarrow u$, w.l.o.g. $\ell(n) \geq k(n)$. Then $\varepsilon_{z u^{-1}} \in \mathcal{S}_{0} \star \mathcal{S}_{0}^{-1}$ and $\varepsilon_{z u^{-1}}=\lim \varepsilon_{\tau^{k(n)}(x)}$ * $\varepsilon_{\tau^{k(n)+(\ell(n)-k(n))}\left(x^{-1}\right)} \in \mathcal{S}$.】

Furthermore, $\mathscr{A}=\operatorname{LIM}\{v(n)\}=\operatorname{LIM}\left\{\varepsilon_{x} \star v^{(1)}(n) \star\right.$ $\left.\varepsilon_{\tau^{n}\left(x^{-1}\right)}\right\}$. Thus $\mathscr{A}=\varepsilon_{x} \star \rho^{(1)} \star \mathcal{S}_{0}^{-1}$.

\section{Continuous Time: Nondissipating Continuous Convolution Semigroups}

Next we replace the random walk by a continuous convolution semigroup $\left\{\lambda_{t}\right\}_{t \in \mathbb{R}_{+}}$(the distribution of a Lévy process on $\mathbb{G}$, if $\mathbb{G}$ is metrizable). In contrast to the discrete time case, now the idempotent $\lambda_{0}=\lim _{t \rightarrow 0} \lambda_{t}$ is uniquely determined.

W.l.o.g. we assume that $\mathbb{G}$ is generated by $\left\{\operatorname{supp}\left(\lambda_{t}\right)\right.$ : $t \geq 0\}$. For short, $\left\{\lambda_{t}\right\}$ is called adapted then. $\left\{\lambda_{t}\right\}_{t \in \mathbb{R}_{+}}$is nondissipating if the concentration functions $f_{\lambda_{t}}(C)$ := $\sup _{x \in \mathbb{G}} \lambda_{t}(C x)$ do not converge to 0 for some compact $C$ (for $t \rightarrow \infty)$.

For any $t_{0}>0$ the random walk $\left\{\lambda_{t_{0}}^{k}=\lambda_{t_{0} k}\right\}$ is called skeleton random walk. Note that a skeleton random walk do not need to be adapted on $\mathbb{G}$ hence we introduce for $t_{0}>0$ the subgroups $\mathbb{G}_{\lambda_{t_{0}}}=: \mathbb{G}_{t_{0}}$ as smallest closed subgroup containing supp $\lambda_{t_{0}}$. Hence $\left\{\lambda_{t_{0}}^{k}\right\}$ is adapted on $\mathbb{G}_{t_{0}}$. We define $N_{t_{0}}:=N_{\lambda_{t_{0}}}$ to be the smallest closed normal subgroup of $\mathbb{G}_{t_{0}}$ containing $\operatorname{supp} \lambda_{t_{0}} * \tilde{\lambda}_{t_{0}}$. Hence $\mathbb{G}_{t_{0}}=N_{t_{0}} \rtimes_{\tau_{t_{0}}} \mathbb{Z}$ and $\lambda_{t_{0} k}=\nu\left(t_{0} k\right) \otimes \varepsilon_{t_{0} k}$. (For later use we identify $\mathbb{Z}$ with $t_{0} \cdot \mathbb{Z}$.)

Again, to avoid trivialities, throughout in the sequel, $\mathbb{G}$ is assumed to be noncompact (else any continuous convolution semigroup would be nondissipating). Hence also the closed subgroups $\mathbb{G}_{t_{0}}$ are noncompact and the results of Section 1 apply. In fact, if for some $t_{0}>0, \mathbb{G}_{t_{0}}$ is compact, then $\left\{\lambda_{t_{0}}^{k}=\right.$ $\left.\lambda_{t_{0} k}: k \in \mathbb{Z}_{+}\right\}$is uniformly tight. But then $\left\{\lambda_{s}: s \geq 0\right\}=$ $\left\{\lambda_{t_{0} k}: k \in \mathbb{Z}_{+}\right\} \star\left\{\lambda_{s}: 0 \leq s \leq t_{0}\right\}$ is uniformly tight. Therefore $\mathbb{G}$ (and all groups $\mathbb{G}_{t}, t>0$ ) are compact.

First we compare the behaviour of concentration functions of continuous convolution semigroups and of their skeleton random walks.

Proposition 12. Let $\left\{\lambda_{t}\right\} \subseteq \mathscr{M}^{1}(\mathbb{G})$ be an adapted continuous convolution semigroup. Then the following assertions are equivalent.

(i) $\left\{\lambda_{t}\right\}$ is nondissipating.

(ii) For all [(ii') some] $t_{0}>0$, the skeleton random walk $\left\{\lambda_{t_{0}}^{k}=\lambda_{k t_{0}}\right\}$ is nondissipating (and adapted on $\left.\mathbb{G}_{t_{0}}\right)$.

(iii) $\left\{\lambda_{t}\right\}_{t \geq 0}$ is relatively (right) shift compact.

(iv) $\left\{\lambda_{t} * \tilde{\lambda}_{t}\right\}_{t \geq 0}$ is relatively compact. (Then $\lim _{t \rightarrow \infty} \lambda_{t} *$ $\widetilde{\lambda_{t}}$ exists.) (v) For all $\left[\left(v^{\prime}\right)\right.$ some $] t_{0}>0, \lambda_{t_{0}}$ (considered as measure on $\left.\mathbb{G}_{t_{0}}\right)$ has finite first-order moments.

(vi) For all [(vi') some] $t_{0}>0, v\left(t_{0}\right)$ (considered as measure on $N_{t_{0}}$ ) has finite logarithmic moments. That is, for all regular $\tau_{t_{0}}$-functions $f$ on $N_{t_{0}}$ one has $\int_{N_{t_{0}}} \log (1+f) \mathrm{d} v\left(t_{0}\right)<\infty$.

The precise formulations of conditions (v) (resp., (vi)) are: for all subadditive functions $f$ on $\mathbb{G}_{t_{0}}$ (resp., for all regular $\tau_{t_{0}}$-functions $g$ on $N_{t_{0}}$ ) we have $\int_{\mathbb{G}_{t_{0}}} f \mathrm{~d} \lambda_{t_{0}}<\infty$ (resp., $\left.\int_{N_{t_{0}}} \log (1+g) \mathrm{d} \nu\left(t_{0}\right)<\infty\right)$. Note that for a subadditive function $f$ on $\mathbb{G}$ the restriction $\left.f\right|_{\mathbb{G}_{t_{0}}}$ is subadditive. However, the sets of subadditive functions defining moments on $\mathbb{G}_{t_{0}}$, might have no subadditive extension to $\mathbb{G}$. Analogously, for $t_{0} \neq t_{1}$, the sets of $\tau_{t_{0}}$ - and $\tau_{t_{1}}$-functions may be different (even if $N_{t_{0}}=N_{t_{1}}$, as will be shown in Proposition 13 later).

Proof. $f_{\alpha * \beta}(C) \leq f_{\alpha}(C)$ for all probabilities $\alpha, \beta$, hence $t \mapsto$ $f_{\lambda_{t}}(C)$ is decreasing. Hence $f_{\lambda_{t}}(C) \nrightarrow 0$ iff inf $\lambda_{t}(C)>0$. Thus, as immediately seen, “(i) $\Leftrightarrow$ (ii) $\Leftrightarrow$ (ii')". And obviously we have "(iii) $\Leftrightarrow$ (iv)" and "(v) $\Rightarrow\left(\mathrm{v}^{\prime}\right)$ ", “(vi) $\Rightarrow$ (vi')". The equivalences "( $\left.\mathrm{ii}^{\prime}\right) \Leftrightarrow\left(\mathrm{v}^{\prime}\right) \Leftrightarrow\left(\mathrm{vi}^{\prime}\right)$ ” (for any fixed $t_{0}>0$ ) follow by Theorem 6 , and hence we also have (ii) $\Leftrightarrow$ (v) $\Leftrightarrow$ (vi). Convergence of $\sigma_{t}:=\lambda_{t} * \widetilde{\lambda_{t}}$ follows by Fact 1(a): For all $t>0 \lim _{k} \sigma_{t k}=: \sigma(t)$ exists. Hence $\sigma(t)=\sigma\left(t^{\prime}\right)$ for commensurable $\left(t, t^{\prime}\right)$, in particular, $\sigma(t)=\sigma(1)$ for all rational $t$. Furthermore, for $f \in C_{0}(\mathbb{G}), t \mapsto\left\langle\sigma_{t}, f\right\rangle$ is uniformly continuous. Whence $\sigma(t)=\sigma(1)$ for all real $t>0$ easily follows.

"(ii') $\Rightarrow$ (iii)". By Fact $1(\mathrm{a})$, (ii $\left.^{\prime}\right)$ is equivalent to shift compactness of $\left\{\lambda_{t_{0}}^{k}\right\}$. Let $x_{k} \in \mathbb{G}$ such that $\left\{\lambda_{t_{0} k} * \varepsilon_{x_{k}^{-1}}\right\}$ is relatively compact. Then, $\left\{\lambda_{t}\right\}$ being a continuous convolution semigroup, the set $\left\{\lambda_{r}: 0 \leq r<t_{0}\right\} *\left\{\lambda_{t_{0} k} * \varepsilon_{x_{k}^{-1}}: k \in \mathbb{Z}_{+}\right\}=$: $\left\{\lambda_{t} \star \varepsilon_{x(t)^{-1}}: t \geq 0\right\}$ is relatively compact (where $x(t):=x_{k}$, if $\left.t_{0} k \leq t<t_{0}(k+1)\right)$. Whence (iii) follows.

"(iii) $\Rightarrow$ (i)". Let $\left\{\lambda_{t} * \varepsilon_{x(t)^{-1}}\right\}$ be relatively compact. Hence for any $\epsilon \in(0,1)$ there exists a compact $C$ such that for all $t \in \mathbb{R}_{+}, \lambda_{t}(C x(t)) \geq \epsilon$. Therefore, $f_{\lambda_{t}}(C)$ fails to converge to 0 .

A priori $N_{t_{0}}:=N_{\lambda_{t_{0}}}$, the smallest closed normal subgroup of $\mathbb{G}_{t_{0}}$ containing supp $\lambda_{t_{0}} * \tilde{\lambda}_{t_{0}}$, might not be normal in $\mathbb{G}$ and might depend on $t_{0}$. In order to apply the results of Section 1 we have to overcome these difficulties.

Proposition 13. Let $\left\{\lambda_{t}\right\}$ be an adapted continuous convolution semigroup. Then one has $N_{t_{0}}=N_{1}=: N$ for all $t_{0}>0$, and $N \triangleleft \mathbb{G}$.

Proof. Obviously, $\mathbb{G}=\left(\bigcup_{t} \mathbb{G}_{t}\right)^{-}$and for all $t>0, \mathbb{G}_{t}=$ $\bigcup_{k \in \mathbb{Z}} N_{t} x_{t}^{k}$ for some (all) $x_{t} \in \operatorname{supp} \lambda_{t}$. First we consider dyadic numbers $t$ and then proceed to real $t$ applying continuity of $\left\{\lambda_{t}\right\}$. 
Let $t_{0}=1$. Then obviously $\mathbb{G}_{1} \subseteq \mathbb{G}_{1 / 2}$ since $\lambda_{1}=\lambda_{1 / 2}^{2}$. For all $x, x_{1 / 2} \in \operatorname{supp} \lambda_{1 / 2}$ it follows, $x x_{1 / 2}, x_{1 / 2} x, x_{1 / 2}^{2} \in \operatorname{supp} \lambda_{1} \subseteq$ $\mathbb{G}_{1}$, hence

$$
\begin{aligned}
x_{1 / 2} x N_{1} & =N_{1} x_{1 / 2} x=x x_{1 / 2} N_{1}=N_{1} x x_{1 / 2} \\
& =N_{1} x_{1 / 2}^{2}=x_{1 / 2}^{2} N_{1} .
\end{aligned}
$$

In fact, $N_{t} \triangleleft \mathbb{G}_{t}$ for all $t>0$, and hence $y N_{t}=N_{t} z$ for all $y, z \in \operatorname{supp} \lambda_{t}$. Therefore,

$$
\begin{aligned}
& x^{-1} x_{1 / 2}^{2} N_{1}=x_{1 / 2} N_{1}, \\
& N_{1} x_{1 / 2}^{2} x^{-1}=N_{1} x_{1 / 2} .
\end{aligned}
$$

Put $N_{1}^{*}:=N_{1} \cap x_{1 / 2} N_{1} x_{1 / 2}^{-1}$, and hence, by (5)

$$
x_{1 / 2} N_{1}^{*}=x_{1 / 2} N_{1} \cap N_{1} x_{1 / 2}=N_{1}^{*} x_{1 / 2} .
$$

Claim 1. We have

$$
\lambda_{1 / 2}\left(N_{1}^{*} x_{1 / 2}\right)=\lambda_{1 / 2}\left(x_{1 / 2} N_{1}^{*}\right)=1 .
$$

By (6) it suffices to show that $\lambda_{1 / 2}\left(x_{1 / 2} N_{1}\right)=$ $\lambda_{1 / 2}\left(N_{1} x_{1 / 2}\right)=1$. According to (4) and (5) we have

$$
\begin{aligned}
1 & =\lambda_{1}\left(N_{1} x_{1 / 2}^{2}\right) \\
& =\int \lambda_{1 / 2}\left(x^{-1} N_{1} x_{1 / 2}^{2}\right) \mathrm{d} \lambda_{1 / 2}(x) \\
& \stackrel{(4)}{=} \int \lambda_{1 / 2}\left(x^{-1} x_{1 / 2}^{2} N_{1}\right) \mathrm{d} \lambda_{1 / 2}(x) \\
& \stackrel{(5)}{=} \int \lambda_{1 / 2}\left(x_{1 / 2} N_{1}\right) \mathrm{d} \lambda_{1 / 2}(x) \\
& =\lambda_{1 / 2}\left(x_{1 / 2} N_{1}\right) .
\end{aligned}
$$

Analogously we have $1=\int \lambda_{1 / 2}\left(N_{1} x_{1 / 2}^{2} x^{-1}\right) \mathrm{d} \lambda_{1 / 2}(x)=$ $\lambda_{1 / 2}\left(N_{1} x_{1 / 2}\right)$. Consequently, supp $\lambda_{1 / 2} \subseteq x_{1 / 2} N_{1}^{*}=N_{1}^{*} x_{1 / 2}$ yields $\mathbb{G}_{1 / 2} \subseteq \bigcup_{k \in \mathbb{Z}} x_{1 / 2}^{k} N_{1}^{*}=\bigcup_{k \in \mathbb{Z}} N_{1}^{*} x_{1 / 2}^{k}$. Thus, as $x_{1 / 2}$ normalizes $N_{1}^{*}$, we obtain $N_{1}^{*} \triangleleft \mathbb{G}_{1 / 2}$.

Claim 2. $N_{1 / 2} \subseteq N_{1}^{*} \subseteq N_{1} . N_{1}^{*} \cap N_{1 / 2}=: N^{*}$ is a closed normal subgroup of $\mathbb{G}_{1 / 2}$ such that $1=\lambda_{1 / 2}\left(x_{1 / 2} N^{*}\right)=\lambda_{1 / 2}\left(N^{*} x_{1 / 2}\right)$. But $N_{1 / 2}$ is minimal with this property. Whence $N_{1 / 2} \subseteq N^{*}$, thus $N_{1 / 2} \subseteq N_{1}^{*} \subseteq N_{1}$.

Claim 3. $N_{1}^{*}=N^{*}=N_{1 / 2}=N_{1}=: N . \llbracket N_{1 / 2} \triangleleft \mathbb{G}_{1 / 2}$ yields $N_{1 / 2} \triangleleft \mathbb{G}_{1}$ (as $\left.G_{1} \subseteq \mathbb{G}_{1 / 2}\right)$. And $\lambda_{1}\left(x_{1 / 2}^{2} N_{1 / 2}\right)=$ $\int \lambda_{1 / 2}\left(x^{-1} x_{1 / 2}^{2} N_{1 / 2}\right) \mathrm{d} \lambda_{1 / 2}(x)=\int \lambda_{1 / 2}\left(x^{-1} x_{1 / 2} N_{1 / 2} x_{1 / 2}\right) \mathrm{d} \lambda_{1 / 2}(x)$; therefore $\lambda_{1}\left(x_{1 / 2}^{2} N_{1 / 2}\right)=\lambda_{1 / 2}\left(N_{1 / 2} x_{1 / 2}\right)=1$ (since $x^{-1} x_{1 / 2} \epsilon$ $N_{1 / 2}$ ). Hence, according to the definition, $N_{1} \subseteq N_{1 / 2}$ follows. Together we obtain $N_{1} \subseteq N_{1 / 2} \subseteq N_{1}^{*} \subseteq N_{1}$, whence the assertion follows.】

By induction, we obtain $N=N_{1 / 2^{n}}$ for all $n \in \mathbb{Z}_{+}$, and $N \triangleleft \bigcup_{k \in \mathbb{Z}, n \geq 0} x_{1 / 2^{n}}^{k} N_{1}$.

Claim 4. $N_{t}=N$ for all $t>0$.
At first we consider dyadic $t=k / 2^{n}$.

(1) $\operatorname{supp} \lambda_{k / 2^{n}} \subseteq\left[\left(x_{1 / 2^{n}} N\right)^{k}\right]^{-}=x_{1 / 2^{n}}^{k} N=N x_{1 / 2^{n}}^{k}$, whence $N_{k / 2^{n}} \subseteq N$. Hence supp $\lambda_{k / 2^{n}} \star \widetilde{\lambda}_{k / 2^{n}} \subseteq N$.

(2) supp $\lambda_{k / 2^{n}} \star \widetilde{\lambda}_{k / 2^{n}} \supseteq x_{1 / 2^{n}}^{k-1} \cdot\left(\operatorname{supp} \lambda_{1 / 2^{n}} \star \tilde{\lambda}_{1 / 2^{n}}\right) \cdot x_{1 / 2^{n}}^{-(k-1)}$. Thus $N_{k / 2^{n}} \supseteq x_{1 / 2^{n}}^{k-1} N x_{1 / 2^{n}}^{-(k-1)}=N$.

Hence $N_{t}=N$ for all dyadic $t>0$. Continuity of $t \mapsto$ $\lambda_{t} \star \tilde{\lambda}_{t}$ easily yields $N_{t} \subseteq N_{1}=N$, and $N \triangleleft\left[\bigcup_{k, n \geq 0} x_{1 / 2^{n}}^{k} N\right]^{-}$.

Replacing 1 by any real $t_{0}>0$ we obtain analogously $N_{t_{0}}=N_{t_{0} k / 2^{n}}$ for $k, n \geq 0$, and again $N_{t} \subseteq N_{t_{0}}$ for all real $t>0$. In particular, $N=N_{1} \subseteq N_{t_{0}}$. Consequently, $N_{t}=N$ for all $t>0$.

Now the assertions follow immediately, and the proof is complete.

Consequently, for any nondissipating continuous convolution semigroup $\left\{\lambda_{t}\right\}$ we obtain that $\left\{\lambda_{t}\right\}$ is adapted iff $\mathbb{G}=$ $\bigcup_{t \geq 0} \mathbb{G}_{t}=\bigcup_{s \in \mathbb{R}} N x_{s}$. Indeed, we have the following.

Proposition 14. Let $\left\{\lambda_{t}\right\}_{t \geq 0}$ be nondissipating and adapted. Then, with the afore-introduced notations, there exists a continuous one-parameter group $\{x(t)\}_{t \in \mathbb{R}} \subseteq \mathbb{G}$ such that $\mathbb{G} / N \cong\{\bar{x}(t): t \in \mathbb{R}\} \cong \mathbb{R}$ and $\operatorname{supp} \lambda_{t} \subseteq N x(t), t \geq 0$.

Let, for $t \in \mathbb{R}, \tau_{t}:=\left.i_{x(t)}\right|_{N}$ denote the restriction of the inner automorphism to $N$. Then $T=\left(\tau_{t}\right)$ is a continuous oneparameter group in $\operatorname{Aut}(N)$. And one has $\mathbb{G} \cong N \rtimes_{T} \mathbb{R}$ (with group operation $\left.(g, t)(h, s)=\left(g \tau_{t}(h), t+s\right), g, h \in N, t, s \in \mathbb{R}\right)$. Furthermore, $\lambda_{t}$ may be represented as $\lambda_{t}=\nu(t) \otimes \varepsilon_{t}$, where $t \mapsto v(t) \in \mathscr{M}^{1}(N)$ is a continuous $M$-semigroup (with respect to $T$ ) (cf. Definition 18 later). Since $x(0)=e$ it follows that $\lambda_{0}=$ $\omega_{H} \otimes \varepsilon_{0}$ for some compact subgroup $H \subseteq N$.

Proof. As shown before, there exists $x_{t} \in \mathbb{G}$ such that $\operatorname{supp} \lambda_{t} \subseteq N x_{t}$ (for all $t \in \mathbb{R}_{+}$). Hence, $\pi: \mathbb{G} \rightarrow$ $\mathbb{G} / N$ denoting the canonical homomorphism, and we obtain $\pi\left(\lambda_{t}\right)=\varepsilon_{\bar{z}(t)}$ with $\bar{z}(t)=x_{t} N$. Hence $\left\{\varepsilon_{\bar{z}(t)}\right\}_{t \in \mathbb{R}_{+}}$and therefore $(\bar{z}(t))_{t \in \mathbb{R}_{+}}$are continuous one-parameter semigroups, extendible to groups, and thus $\mathbb{G} / N \cong \mathbb{R}$. 【Indeed, if $\mathbb{G} / N$ were compact, $\mathbb{G}_{t_{0}} / N$ would be compact (hence finite) for any $t_{0}$, a contradiction to Fact 1(b). 』 Finally, there exists a continuous one-parameter group $(x(t))_{t \in \mathbb{R}}$ in $\mathbb{G}$ with $\pi(x(t))=\bar{z}(t), t \geq 0$, (cf., e.g., [41]), whence the assertion follows.

To show that $\mathbb{G}$ splits as a semi-direct product, put $N \cap$ $X=: L$, where $X:=\{x(t)\}_{t \in \mathbb{R}}$. Assume $L$ to be nontrivial. Then $X / L$ is compact, hence $\mathbb{G} / N=(N \cdot X) / N$ is compact, in contradiction to $\mathbb{G} / N \cong \mathbb{R}$, as shown previously.

Hence $\mathbb{U}:=\bigcup_{t} \mathbb{G}_{t}=N \rtimes_{T} \mathbb{R}$ is closed in $\mathbb{G}$ containing $\bigcup_{t} \operatorname{supp} \lambda_{t}$, whence $\mathbb{G}=\mathbb{H}$.

The existence of nondissipating adapted continuous convolution semigroups $\left(\lambda_{t}\right)$ has strong influence on the structure of $\mathbb{G}$.

Corollary 15. Assume, as in Proposition 14, $\left(\lambda_{t}\right)$ to be adapted and nondissipating, and assume in addition $N$ to be totally disconnected. Then $\mathbb{G}=N \otimes \mathbb{R}, N$ is compact and the $M$ semigroup $(\nu(t))$ is a continuous convolution semigroup. If 
moreover $\mathbb{G}$ is assumed to be totally disconnected then $\mathbb{G}=N$, hence $\mathbb{G}$ is compact, the trivial case excluded afore.

【If $N$ is totally disconnected then the continuous automorphism group $T=\left\{\left.i_{x(t)}\right|_{N}\right\}$ is trivial; hence $N \rtimes_{T} \mathbb{R}=$ $N \otimes \mathbb{R}$. Since $T$ is trivial, any continuous $M$-semigroup (with respect to $T$ ) is a continuous convolution semigroup. Thus we obtain immediately that $N$ is compact if $\left(\lambda_{t}\right)$ is nondissipating.

If moreover, $\mathbb{G}$ is totally disconnected, then $\{x(t)\}$ is trivial, whence $\mathbb{G}=N$ is compact. $\rrbracket$

In the discrete time case it was essentially used that $N \cong$ $C_{K}(\tau)$. As mentioned in the previous proof, in the continuous time case, for all $t>0$, we have $N=C_{K(t)}$. It turns out (cf. the next proposition) that $K(t)$ is independent of $t>0$. Furthermore, in the continuous time case the structure of $N$ is nicer. Put $C:=C(T)=\left\{x \in N: \tau_{t}(x) \stackrel{t \rightarrow \infty}{\longrightarrow} e\right\}$ and $C_{K}(T)=\left\{x \in N: \tau_{t}(x) \stackrel{t \rightarrow \infty}{\longrightarrow} e \bmod K\right\}$ for some compact, $T$-invariant subgroup $K$.

Proposition 16. Let, as before, $\mathbb{G}=N \rtimes_{T} \mathbb{R}$.

(a) With the notations of Proposition 14 one has $N \cong$ $C_{K}(T)$.

In fact, for any locally compact group $N$, admitting a continuous one parameter group $T=\left(\tau_{t}\right) \subseteq \operatorname{Aut}(N)$ and $a$ $T$-invariant compact subgroup $K$ one has the following.

(b) For all $t>0, C_{K}(T)=C_{K}\left(\tau_{t}\right)$, in particular, $C(T)=C\left(\tau_{t}\right)$.

(c) $C(T)$ is closed, T-invariant, connected, and isomorphic to a contractible Lie group (hence a homogeneous group), and $C_{K}(T)$ is a closed $T$-invariant subgroup of $N$. If $N$ is compact, then $C_{K}(T)=K$.

(d) $C(T) \triangleleft C_{K}(T)$, and there exists a continuous homomorphism $\beta$ : Aut $(K) \rightarrow \operatorname{Aut}(C(T)), \beta(\kappa)(g):=$ $\kappa g \kappa^{-1}, \kappa \in K, g \in C(T)$, such that $C_{K}(T)=C(T) \rtimes_{\beta} K$. In particular, $C_{K}(T)$ is compactly generated.

(e) The restrictions $S:=\left.T\right|_{C(T)}=\left(\sigma_{t}\right)_{t \in \mathbb{R}} \subseteq$ $\operatorname{Aut}(C(T))$ and $\Gamma:=\left.T\right|_{K}=\left(\gamma_{t}\right)_{t \in \mathbb{R}} \subseteq \operatorname{Aut}(K)$ are continuous one-parameter groups, with $\Gamma$ consisting of inner automorphisms of $K$.

(f) $T=\left(\tau_{t}=\left(\sigma_{t}, \gamma_{t}\right)\right)$ satisfies the following consistency conditions $\sigma_{t} \circ \beta(\kappa)=\beta\left(\gamma_{t}(\kappa)\right) \circ \sigma_{t}, \kappa \in K, t \in \mathbb{R}$.

Conversely, any group $T$ arises in that way.

Proof. (a) As mentioned in the previous proof, for any $t>0$, we have $N=C_{K(t)}\left(\tau_{t}\right), K(t)$ denoting a compact $\tau_{t}$-invariant subgroup $K(t)$.

It follows easily that $K(t)=K(s)=: K$ for all $t, s>0$. Indeed:

(1) For $t>0, N \in \mathbb{N}$, it follows that $K(t / N)=$ $K(t)$. «Obviously, $K(t) \subseteq K(t / N)$. On the other hand, $K(t / N)$ is compact and $\tau_{t / N}^{k}$-invariant for all $k \in \mathbb{Z}$, in particular, $K(t / N)$ is $\tau_{t}$-invariant. As $\tau_{t}$ is compactly contracting $\bmod K(t)$, we obtain
$K(t / N)=\tau_{t / N}^{n \cdot N}(K(t / N))=\tau_{t}^{n}(K(t / N)) \rightarrow e \bmod$ $K(t)$, whence $K(t / N) \subseteq K(t)$ follows. \

(2) Let $t, s>0$. Then there exists a sequence $k(n) \rightarrow \infty$ such that $n \cdot s=k(n) \cdot t+r_{n}$ with $0 \leq r_{n}<t$. For $x \in N=$ $C_{K(t)}\left(\tau_{t}\right)$ we have $\tau_{t}^{n}(x)=y_{n}^{t} \kappa_{n}^{t}$ with $y_{n}^{t} \rightarrow e$ and $\kappa_{n}^{t} \epsilon$ $K(t)$. Hence $\tau_{s}^{n}(x)=\tau_{r_{n}}\left(y_{n}^{t}\right) \tau_{r_{n}}\left(\kappa_{n}^{t}\right)$. The first terms converge to $e$, and $\operatorname{LIM}\left(\tau_{r_{n}}\left(\kappa_{n}^{t}\right)\right) \subseteq \bigcup_{0 \leq u \leq t} \tau_{u}(K(t))$. Hence $\operatorname{LIM}\left(\tau_{s}^{n}(x)\right) \subseteq K(s)$ (since $N=C_{K(s)}\left(\tau_{s}\right)$ ), on the other hand, $\operatorname{LIM}\left(\tau_{s}^{n}(x)\right) \subseteq \bigcup_{0 \leq u \leq t} \tau_{u}(K(t))$.

Replacing $t$ by $t / N, N \in \mathbb{N}$, in view of Step (1). we obtain $K(s) \subseteq \bigcup_{0 \leq u \leq t / N} \tau_{u}(K(t))$. Continuity of $u \mapsto \tau_{u}$ finally yields $K(s) \subseteq K(t)$. And analogously, $K(t) \subseteq K(s)$. Whence $K(t)=$ : $K$ does not depend on $t>0$. According to (b), we obtain $C_{K}\left(\tau_{t}\right)=C_{K}(T)$. Thus (a) is proved.

(b) See [2], [6, Lemma 3.2.26]. (Note that the group $\left\{\tau_{t}\right\}_{t>0}$ is multiplicatively parametrized.) (c), (d) see [6, Theorem 3.2.32], [4]. For (e) see [5, Lemma 3.3], [6], and for (f) see $[6,3.3 .4],[4]$.

For later use (in the proof of Theorem 22 and in Lemmas A.1-A.5) we mention the following.

Proposition 17. With the notations introduced afore (in Proposition 16) one has the following. There exists a continuous one-parameter subgroup $Y=(y(t))_{t \in \mathbb{R}}$ such that for all $t, y(t)=\kappa(t) x(t)$ for some $\kappa(t) \in K$-hence in particular, $N x(t)=N y(t)$-and $Y$ commutes element wise with $K$.

Consequently, the automorphism group $\bar{T}:=\left(\bar{\tau}_{t}:=\left.i_{y(t)}\right|_{N}\right)$ belongs to the centralizer $\operatorname{Cent}(\beta(K), \operatorname{Aut}(N))$.

Proof. $T$ is contracting mod. $K, X=:(x(t)) \cong \mathbb{R}$ is closed and $K$ is $T$-invariant. Therefore, $\Gamma:=K \cdot X$ is a closed subgroup of $\mathbb{G}$, representable as semidirect product $\Gamma=K \rtimes_{T} \mathbb{R}$. According to [42, Proposition 9.4] or [43, Proposition 1.24], there exists a subgroup $Y=(y(t)) \cong X(\cong \mathbb{R}), Y \triangleleft \Gamma$, such that $\Gamma=K \otimes Y$. That is, $y(t)=\kappa(t) x(t)$ for some $\kappa(t) \in K$ and $y(t) \kappa=\kappa y(t)$ for all $t$ and all $\kappa \in K$.

In the sequel, in the proof of Theorem 20, respectively, in Lemmas A.1-A.5 we will always assume that $X=Y$ and $T=$ $\bar{T}$.

Now we define in analogy to the discrete time case the following.

Definition 18. Let $N$ be a locally compact group, $T=\left(\tau_{t}\right) \subseteq$ $\operatorname{Aut}(N)$ as before.

(a) $\{\nu(t)\}_{t \in \mathbb{R}_{+}} \subseteq \mathscr{M}^{1}(N)$ is a (continuous time) M-semigroup (with respect to $T=\left(\tau_{t}\right)$ )-also called skew convolution semigroup, $T$-semigroup, or distribution of a generalized Ornstein-Uhlenbeck process-if $t \mapsto v(t)$ is continuous and if the following cocycle equation is satisfied:

$$
\nu(t+s)=v(t) \star \tau_{t}(\nu(s)) \quad \forall t, s \in \mathbb{R}_{+} .
$$

In the following we are interested in relatively compact M-semigroups. 
(b) $\rho \in \mathscr{M}^{1}(N)$ is $T$-decomposable (or $T$-self-decomposable), if for all $t \in \mathbb{R}_{+}, \rho=\nu(t) \star \tau_{t}(\rho)$. Again, Cof ${ }_{\rho}\left(\tau_{t}\right)$ denotes the set of cofactors. $\rho \in \mathscr{M}^{1}(N)$ is $K$ $T$-decomposable if in addition, $\rho$ is right $K$-invariant.

In analogy to Remark 5 we note the following.

Remark 19. Let $N$ be a locally compact group, $T \subseteq \operatorname{Aut}(N)$. Let $\left\{\lambda_{t}=\nu(t) \otimes \varepsilon_{t}\right\}$ be a space-time continuous convolution semigroup on $\mathbb{G}=N \rtimes_{T} \mathbb{R}$. If $\left\{\lambda_{t}\right\}$ is not adapted then there exists a closed subgroup $\mathbb{G}^{\prime}=\bigcup_{t} N^{\prime} \cdot(z(t), t)$ on which it is adapted. $N^{\prime} \subseteq N$ is a closed subgroup and $(y(t)=(z(t), t))$ denotes a continuous one-parameter group in the normalizer of $N^{\prime}$. Furthermore, $\lambda_{t}=\left(\nu^{\prime}(t) \otimes \varepsilon_{0}\right) * \varepsilon_{(z(t), t)}=\left(\nu^{\prime}(t) \star \varepsilon_{z(t)}\right) \otimes \varepsilon_{t}$ where $\left\{\nu^{\prime}(t)\right\}$ is a continuous M-semigroup with respect to $T^{\prime}=\left(\tau_{t}^{\prime}\right)$, with $\left(\tau_{t}^{\prime}:=\left.i_{y(t)}\right|_{N^{\prime}}=i_{z(t)} \circ \tau_{t}\right)$.

Therefore, $\left\{\lambda_{t}\right\}$ is adapted for all closed subgroups $N^{\prime} \subseteq$ $N$ and all continuous one-parameter groups $((z(t), t))$ in the normalizer of $N^{\prime}$, satisfying for all $t \in \mathbb{R}$

$$
\begin{aligned}
& \text { (1) } \tau_{t}\left(N^{\prime}\right)=i_{z(t)^{-1}}\left(N^{\prime}\right), \\
& \text { (2) } \operatorname{supp} v(t) \subseteq N^{\prime} z(t) \Longrightarrow N^{\prime}=N .
\end{aligned}
$$
(d).

We have the continuous time analogues of Theorem 6(a)-

Theorem 20. Let, as before (Proposition 16), $\mathbb{G}=N \rtimes_{T} \mathbb{R}, \lambda_{s}=$ $\nu(s) \otimes \varepsilon_{s}, s \geq 0$, be probabilities on the on the space-time building $\mathbb{G} \cong N \rtimes_{T} \mathbb{R}$. Obviously, $\left\{\lambda_{t}\right\}$ is a continuous convolution semigroup iff $\{\nu(t)\}$ is a continuous $M$-semigroup with respect to T. $\lambda_{0}=\lim _{t \rightarrow 0} \lambda_{t}$ is idempotent and $\lambda_{0} * \lambda_{t} * \lambda_{0}=\lambda_{t}$, for all $t$, hence $\nu(0)=\omega_{H}$ with $\omega_{H} \star \nu(t) \star \tau_{t}\left(\omega_{H}\right)=\nu(t), t \geq 0$, and $\nu(0)=\lim _{t \rightarrow 0} \nu(t)$. (H denotes a compact subgroup of $N$.)

(a) If $\left\{\lambda_{t}\right\}$ is nondissipating and adapted then $\{\nu(t)\}$ is relatively compact and satisfies condition (10). Furthermore, $N=C_{K}(T)$ for some compact $T$-invariant subgroup $K \subseteq N$.

(b) Conversely, if $\{\nu(t)\}$ is relatively compact and satisfies condition (10) then the continuous convolution semigroup $\left\{\lambda_{t}\right\}$ is nondissipating and adapted. Hence (a) applies.

(c) In (a), respectively, (b), $\lim _{t \rightarrow \infty} \nu(t) \star \omega_{K}=: \rho \in$ $\mathscr{M}^{1}(N)$ exists and is T-K-decomposable with cofactors $\{v(t)\}$.

(d) Conversely, let $N=C_{K}(T)$ and let $\rho \in \mathscr{M}^{1}(N)$ be $K-T$-decomposable. Then there exists a nondissipating continuous convolution semigroup $\left\{\lambda_{t}=\nu(t) \otimes\right.$ $\left.\varepsilon_{t}\right\} \subseteq \mathscr{M}^{1}(\mathbb{G})$, with a relatively compact continuous $M$ semigroup $\{\nu(t)\}$ in $\mathscr{M}^{1}(N)$ such that $\nu(t) \in \operatorname{Cof}_{\rho}\left(\tau_{t}\right)$ for all $t$, and furthermore, $\rho=\lim _{t \rightarrow \infty} \nu(t) \star \omega_{K}$.

Proof. (a) is proved as in the discrete time case, Theorem 6(d). (For aperiodic groups see [22, Proposition A], [23, Theorem 3.16]).

(b) and (c) are proved analogous to Theorem 6(b) and (c).

(d) Let $\rho \in \mathscr{M}^{1}(N)$ be $K-T$-decomposable. Again as in the case of discrete times, Theorem $6(\mathrm{~d})$, for any $t>0$, there exists a relatively compact (discrete) M-semigroup $\left\{v_{t}(k)\right\}_{k \geq 0}$ of cofactors (with respect to $\left(\tau_{t}^{k}\right)$ ), such that $\lim _{k} v_{t}(k) \star \omega_{K}=$ $\rho$. In fact, for any selection $\bar{\nu}(t) \in \operatorname{Cof}_{\rho}\left(\tau_{t}\right), t \geq 0$, it follows as in Theorem $6(\mathrm{~d})$ that $\tau_{t}(\rho) \rightarrow \omega_{K}$ and $\bar{\nu}(t) \star$ $\omega_{K} \rightarrow \rho$. We have to show that the cofactors $\{\bar{\nu}(t)\}$ can be chosen as continuous M-semigroup in $\mathscr{M}^{1}(N)$. For this purpose we need some tools from the embedding problem for infinitely divisible laws on groups. Recall that $\{\nu(t)\}$ is a continuous M-semigroup iff $\left\{\lambda_{t}=\nu(t) \otimes \varepsilon_{t}\right\}$ is a continuous convolution semigroup (in $\mathscr{M}^{1}(\mathbb{G})$ ). So we have to prove the existence of $\lambda=\lambda_{1} \in \mathscr{M}^{1}(\mathbb{G})$, embeddable into a continuous convolution semigroup $\left(\lambda_{t}=\nu(t) \otimes \varepsilon_{t}\right)$ such that $\nu(t) \epsilon$ $\operatorname{Cof}_{\rho}\left(\tau_{t}\right), t \geq 0$ (cf., for aperiodic groups, in particular simply connected nilpotent Lie groups, [23, Proposition 3.6]; see also [22, Theorem B]).

Claim 1. There exist infinitely divisible $\lambda=\lambda_{1}=\nu \otimes \varepsilon_{1}$, $\nu \in \operatorname{Cof}_{\rho}\left(\tau_{1}\right)$ with roots $\lambda_{1 / m}=\nu(1 / m) \otimes \varepsilon_{1 / m}$ where $\nu(1 / m) \epsilon$ $\operatorname{Cof}_{\rho}\left(\tau_{1 / m}\right)$ and $\nu=\star_{0}^{m-1} \tau_{j / m}(\nu(1 / m))$. Furthermore, $\lambda$ is embeddable into a rational convolution semigroup $\left\{\lambda_{r}\right\}_{r \in \mathbb{Q}_{+}}$. Moreover, the root sets $\mathscr{R}_{1 / m}:=\left\{\sigma \in \mathscr{M}^{1}(\mathbb{G}): \sigma^{m}=\lambda\right\}$ are compact and consist of measures $\sigma=\mu \otimes \varepsilon_{1 / m}$, with $\mu \in \operatorname{Cof}_{\rho}\left(\tau_{1 / m}\right)$.

$\llbracket$ Choose, for $n \in \mathbb{N}, \bar{\nu}(1 / n) \in \operatorname{Cof}_{\rho}\left(\tau_{1 / n}\right)$, put $\nu^{(n)}:=$ $\star_{0}^{n-1} \tau_{j / n}(\bar{\nu}(1 / n))$; hence $\nu^{(n)} \in \operatorname{Cof}_{\rho}\left(\tau_{1}\right)$ for all $n$. Put $\lambda_{1 / n}^{(n)}:=$ $\bar{\nu}(1 / n) \otimes \varepsilon_{1 / n}$; hence $\lambda^{(n)}:=\left(\lambda_{1 / n}^{(n)}\right)^{n}=\left(\bar{\nu}(1 / n) \otimes \varepsilon_{1 / n}\right)^{n}=$ $\nu^{(n)} \otimes \varepsilon_{1}$.

According to the shift-compactness theorem, $\operatorname{Cof}_{\rho}\left(\tau_{t}\right)$ is compact for all $t>0$. For $n \geq m$, put $\bar{\lambda}_{1 / m}^{(n)}:=\left(\lambda_{1 / n !}^{(n !)}\right)^{(n ! / m)}=$ : $\bar{\nu}^{(n)}(1 / m) \otimes \varepsilon_{1 / m}\left(\right.$ with $\left.\bar{\nu}^{(n)}(1 / m) \in \operatorname{Cof}_{\rho}\left(\tau_{1 / m}\right)\right)$, and $\bar{\lambda}_{1}^{(n)}=$ $\lambda^{(n)}$.

By Tychonoff's theorem, there exists a subnet $\left(n^{\prime}\right) \subseteq \mathbb{N}$ satisfying $\bar{\nu}^{(n)}(1 / m) \stackrel{\left(n^{\prime}\right)}{\longrightarrow} v(1 / m) \in \operatorname{Cof}_{\rho}\left(\tau_{1 / m}\right)$, equivalently, $\bar{\lambda}_{1 / m}^{(n)} \stackrel{\left(n^{\prime}\right)}{\longrightarrow} \lambda_{1 / m}:=\nu(1 / m) \otimes \varepsilon_{1 / m}$, for all $m \in \mathbb{N}$. Continuity of convolution yields $\lambda_{1 / m}^{m}=\lambda_{1}=\lambda=\nu(1) \otimes \varepsilon_{1}, \nu(1) \in \operatorname{Cof}_{\rho}\left(\tau_{1}\right)$ (for all $m \in \mathbb{N}$ ).

We have shown that $\lambda=\lambda_{1}$ is infinitely divisible, and by construction, the roots satisfy $\left(\lambda_{1 / m !}\right)^{m}=\lambda_{1 /(m-1) !}$; hence, $\lambda$ is embeddable into a rational convolution semigroup $\left\{\lambda_{r}\right\}_{r \in \mathbb{Q}}$, (cf. [36, 44]), and the construction easily yields moreover $\lambda_{r}=\nu(r) \otimes \varepsilon_{r}$, with $\nu(r) \in \operatorname{Cof}_{\rho}\left(\tau_{r}\right), r \geq 0$.

Let $\sigma \in \mathscr{M}^{1}(\mathbb{G})$ with $\sigma^{m}=\lambda=\nu \otimes \varepsilon_{1}$. Then, as easily seen, $\sigma=\mu \otimes \varepsilon_{1 / m}$ with $\star_{0}^{m-1} \tau_{j / m}(\mu)=\nu$. Hence, $\mu \in \operatorname{Cof}_{\rho}\left(\tau_{1 / m}\right)$. Therefore, in particular, $\mathscr{R}_{1 / m}$ is compact for all $m$.

In order to show that a continuous convolution semigroup $\left\{\lambda_{\mathrm{t}}^{\prime}\right\}$ can be selected, put $\mathscr{R}:=\bigcup_{n \geq 1}\left\{\sigma_{n}^{m}: \sigma_{n}^{n}=\lambda, 0 \leq\right.$ $m \leq n\}$. As mentioned previously, $\sigma_{n}=\mu_{n} \otimes \varepsilon_{1 / n}$ and $\sigma_{n}^{m}=$ $\star_{0}^{m-1} \tau_{j / n}\left(\mu_{n}\right)$.

Claim 2. $\mathscr{R}$ is relatively compact in $\mathscr{M}^{1}(\mathbb{G})$ with all accumulation points of the form $\nu(t) \otimes \varepsilon_{t}, \nu(t) \in \operatorname{Cof}_{\rho}\left(\tau_{t}\right), 0 \leq t \leq 1$.

【Let $t_{k}:=m_{k} / n_{k} \in[0,1]$ with $m_{k}, n_{k} \in \mathbb{N}, t_{k} \rightarrow$ $t \in[0,1]$. Let $\sigma_{n_{k}}=\mu_{n_{k}} \otimes \varepsilon_{1 / n_{k}} \in \mathscr{R}$, and put 
$\nu\left(t_{k}\right):=\star_{j=0}^{m_{k}} \tau_{j / n_{k}}\left(\mu_{n_{k}}\right)$. Then $\tau_{t_{k}}(\rho) \rightarrow \tau_{t}(\rho)$ and $\rho=\nu\left(t_{k}\right) \star$ $\tau_{t_{k}}(\rho)$ yield that $\left\{\nu\left(t_{k}\right)\right\}$ is relatively compact and $\operatorname{LIM}\left\{\nu\left(t_{k}\right)\right\} \subseteq$ $\operatorname{Cof}_{\rho}\left(\tau_{t}\right)$.】

Let $\mathscr{A}:=\operatorname{LIM}\left\{\lambda_{t}: t \in \mathbb{Q}_{+} \cap[0,1], t \rightarrow 0\right\}$. Therefore, $\sigma \in \mathscr{A}$ is representable as $\sigma=u \otimes \varepsilon_{0}$ for $u \in \operatorname{Cof}_{\rho}\left(\tau_{0}\right)$, with $\tau_{0}=i d$; hence $u \star \rho=\rho$. And, by definition of $\mathscr{A}$, it follows that $\sigma * \lambda_{t}=(u \star \nu(t)) \otimes \varepsilon_{t}, \lambda_{t} * \sigma=\left(\nu(t) \star \tau_{t}(u)\right) \otimes \varepsilon_{t}$ for all $t \geq 0$.

Siebert's embedding theorem (see $[44,5$. Satz 1, 6. Satz 1], [36, Theorem 3.5.4]) shows the existence of a continuous convolution semigroup $\left\{\lambda_{t}^{\prime}\right\}$ of the form $\lambda_{t}^{\prime}=\nu^{\prime}(t) \otimes \varepsilon_{t}$ for all $t$, with $\lambda_{t}^{\prime}=\sigma_{t} * \lambda_{t}=\lambda_{t} * \sigma_{t}$ where $\sigma_{t} \in \mathscr{A}$, hence $\sigma_{t}=u_{t} \otimes \varepsilon_{0}$, $u_{t} \in \operatorname{Cof}_{\rho}\left(\tau_{0}\right)$. In particular, $\nu^{\prime}(t)=\nu(t) \star \tau_{t}\left(u_{t}\right)$. Hence, as immediately seen, $\nu^{\prime}(t) \in \operatorname{Cof}_{\rho}\left(\tau_{t}\right)$.

Since $\left\{\lambda_{t}^{\prime}\right\}$ is a continuous convolution semigroup, $\left\{v^{\prime}(t)\right\}$ is a continuous M-semigroup of cofactors of $\rho$. Whence the assertion. tions.

For the following Theorem 22 we need some prepara-

Let $\mathbb{W}$ be a locally compact group and $\left\{\lambda_{t}\right\}$ a continuous convolution semigroup. The Lévy measure $\eta$ is defined by $\int g \mathrm{~d} \eta=\mathrm{d}^{+} /\left.\mathrm{d} t\right|_{t=0} \int g \mathrm{~d} \lambda_{t}$, for $g \in C^{b}(\mathbb{U})$ vanishing in a neighbourhood of the unit. If as before, $\mathbb{H}=\mathbb{G}=N \rtimes_{T} \mathbb{R}$ and $\lambda_{t}=\nu(t) \otimes \varepsilon_{t}, t \geq 0$, then, as easily seen, the Lévy measure $\eta$ is supported by $N$ and $\int_{N} g \mathrm{~d} \eta=\mathrm{d}^{+} /\left.\mathrm{d} t\right|_{t=0} \int_{N} g \mathrm{~d} \nu(t)$. Recall that Lévy measures are bounded outside neighbourhoods of e. We recall a result of Siebert (see [45, Theorem 1], [46, Theorem 5]).

Fact 3. Let $g \geq 1$ be a submultiplicative function. Let $\epsilon>1$, such that $U=U_{g, \epsilon}=\{g \leq \epsilon\} \in \mathfrak{U}_{\mathbb{\natural}}$. Then we have

$$
\int g \mathrm{~d} \lambda_{t}<\infty \text { for some [all] } t \geq 0 \text { iff } \int_{\mathbb{G} \mid U} g \mathrm{~d} \eta<\infty \text {. }
$$

Thus for subadditive functions $f \geq 0$ we have with $g:=1+f$ : $\int 1+f \mathrm{~d} \lambda_{t}<\infty$ iff $\int_{\mathbb{G} \backslash U} 1+f \mathrm{~d} \eta<\infty$. Equivalently, $\int f \mathrm{~d} \lambda_{t}<$ $\infty$ iff $\int_{\mathbb{G} \backslash U} f \mathrm{~d} \eta<\infty$.

As mentioned before, if the integral on the right side of (11) is finite for $U=U_{g, \epsilon}$, then it is finite for any $U \in \mathfrak{U}_{\mathbb{\square}}$. And, if it is finite for some relatively compact $U_{0} \in \mathfrak{U}_{\mathbb{\square}}$, it is finite for all $U \in \mathfrak{U}_{\mathbb{H}}$.

The following Steps 1-5 are more or less folklore (though it seems to be hard to find precise references), respectively, they are easy generalizations of known results. Therefore we postpone the proofs to the Appendix (as Lemmas A.1 to A.5). Let throughout in the sequel $\mathbb{G}=N \rtimes_{T} \mathbb{R}$ with $N=C_{K}(T)$. Recall (cf. Proposition 16) that we have $N=$ $C \rtimes_{\beta} K$, where $C=C(T)$ is a homogeneous group, that is, a contractible simply connected nilpotent Lie group, and $T=\left(\tau_{t}=\left.i_{x(t)}\right|_{N}\right)_{t \in \mathbb{R}}$. We will tacitly assume, according to Proposition 17, that $\{x(t)\}_{t \in \mathbb{R}}$ and $K$ commute element wise. Recall that a homogeneous group norm (adapted to $T$ ) is a continuous subadditive function $C(T) \rightarrow \mathbb{R}_{+}$satisfying $\|x\|=0$ iff $x=e,\|x\|=\left\|x^{-1}\right\|$ and $\left\|\delta_{t}(x)\right\|=t \cdot\|x\|$, where $\left(\delta_{t}\right)$ denotes a group of dilations defined by $T$ (cf. for example, [6, 2.1.10, 2.7.26 (d)], [29]).

Step 1 (cf. Lemma A.1). There exists a homogeneous norm $\|\cdot\|$ on $C$ which is in addition $\beta(K)$-invariant.

Step 2 (cf. Lemma A.2). Define the function $\phi: N=$ $C \rtimes_{\beta} K \rightarrow \mathbb{R}_{+}$by $\phi(x, \kappa):=\|x\|,\|\cdot\|$ as in Step 1 . Then $\phi$ is subadditive, continuous and satisfies $\phi(\gamma)=\phi\left(\gamma^{-1}\right), \phi(\gamma)=0$ iff $\gamma=(e, \kappa) \in K$ and furthermore, $\phi$ is $K$-bi-invariant. (We call $\phi$ pseudo-group norm adapted to $T$.)

Moreover, for any $t_{0}>0$ there exist constants $0<r \leq$ $R \leq 1,0<s \leq S<1$, satisfying for all $\gamma \in N$ the growth conditions

$$
\begin{array}{ll}
r^{n} \phi(\gamma) \leq \phi\left(\tau_{t_{0}}^{n}(\gamma)\right) \leq R^{n} \phi(\gamma), & n \geq 0, \\
S^{n} \phi(\gamma) \leq \phi\left(\tau_{t_{0}}^{n}(\gamma)\right) \leq s^{n} \phi(\gamma), & n<0 .
\end{array}
$$

For large $t_{0}$ one can put for example, $R=\left\|\left.\tau_{t_{0}}\right|_{C}\right\|, r:=$ $\left\|\left.\tau_{t_{0}}^{-1}\right|_{C}\right\|^{-1}, S=R, s=r$. The automorphism norm is defined as $\|\sigma\|:=\sup \{\|\sigma(x)\|: x \in C,\|x\|=1\}$. In particular, $\left\|\left.\tau_{s+t}\right|_{C}\right\| \leq\left\|\left.\tau_{t}\right|_{C}\right\| \cdot\left\|\left.\tau_{s}\right|_{C}\right\|,\left\|\left.\tau_{t}\right|_{C}\right\| \rightarrow 0$ for $t \rightarrow \infty$ and $\left\|\left.\tau_{-t}\right|_{C}\right\| \geq 1$ for $t \geq 0$.

Step 3 (cf. Lemma A.3). Let $f: N \rightarrow \mathbb{R}_{+}$be a regular $\tau_{t_{0}}$ function for some $t_{0}>0, f\left(\tau_{t_{0}}^{p}(\gamma)\right)=\alpha f(\gamma), 0<\alpha<1$. (See Definition 1.)

Then we obtain $\log (1+f) \sim \log (1+\phi)$, $(\phi$ defined in Step 2.)

Remark 21. As mentioned before, in the situation $N=$ $C$, a contractible (nilpotent) Lie group, it follows that the definition of finite logarithmic moments via group norms (e.g., in $[6,29,30]$ ) and Definition $1(d)$ coincide. Note that $\|\cdot\|$ and $\phi$ are not $\tau_{t}$-functions-they are $\delta_{t}$-functions for dilations $\delta_{t}$, as the proof shows-but these functions have similar properties (cf. (12)).

Step 4 (cf. Lemma A.4). With the notations of the preceding Steps we have: the functions $\psi: \mathbb{G}=N \rtimes_{T} \mathbb{R} \rightarrow \mathbb{R}_{+}$, $\psi(\gamma, t):=1+\phi(\gamma)+\left\|\tau_{-|t|}||_{C}\right\|$ is continuous, submultiplicative, $\geq 2$ and satisfies $\psi(\gamma, t)=2$ iff $t=0, \gamma \in K$. Therefore $\log \psi$ is subadditive and $>0$. Moreover $\{\psi<\alpha\}$ is relatively compact and belongs to $\mathfrak{U}_{\mathbb{G}}$ for all $\alpha>2$.

Step 5 (cf. Lemma A.5). Let, as before, $N=C \rtimes_{\beta} K$ and $\mathbb{G}=N \rtimes_{T} \mathbb{R} . N$ and $\mathbb{G}$ are compactly generated for any neighbourhood $U \in \mathcal{U}_{C}, V:=U \cdot K$ generates $N$, and, for example, for any $\epsilon>0, W:=V \otimes[-\epsilon, \epsilon]$ generates $\mathbb{G}$.

Let $f$ be a regular $\tau_{t_{0}}$-function, for some $t_{0}>0$. Let, for $t>0, W_{t}:=\bigcup_{0 \leq s \leq t}\left\{V \otimes\{s\} \cup\{V \otimes\{s\}\}^{-1}\right\}$ where $V=\{f \leq$ $1\}$. Then $W_{t}$ generates $\mathbb{G}$, and for any symmetric relatively compact $W \in \mathcal{U}_{\mathbb{G}}$ with $W=W K$, we have $\delta_{W_{t}} \sim \delta_{W}$. (For the definition of $\delta_{W}$ cf. (1).) In particular, for all $t_{1}, t_{2}>0$, it follows that $\delta_{W_{t_{1}}} \sim \delta_{W_{t_{2}}}$.

Next we characterize-in analogy to Theorem 6(e)nondissipativity by moment conditions. For the continuous 
time case we introduce additional equivalent conditions $\left(b^{*}\right)$, $\left(d^{*}\right),\left(e^{*}\right)$ which depend on $T$ but not on the particular automorphisms $\tau_{t_{0}}$.

Theorem 22. Let, as before, $\mathbb{G}=N \rtimes_{T} \mathbb{R}$ with $N=C_{K}(T)=$ $C \rtimes_{\beta} K, C=C(T)$ a homogeneous group. Let $\left\{\lambda_{t}=\nu(t) \otimes \varepsilon_{t}\right\}_{t \geq 0}$ be an adapted continuous convolution semigroup with Lévy measure $\eta$. Let $\phi$ denote the pseudo-group norm on $N$ introduced in Step 2 via the K-invariant homogeneous norm $\|\cdot\|$ on $C=C(T)$ (cf. Step 1). Then the following assertions are equivalent.

(a) $\left\{\lambda_{t}\right\}$ is nondissipating.

(b) For some (any) $t_{0}>0, \lambda_{t_{0}}$ has finite first-order moments (on $\mathbb{G}$ ).

$\left(\mathrm{b}^{*}\right)$ For some (any) $t_{0}>0, \int_{\mathbb{G}} \delta_{W} \mathrm{~d} \lambda_{t_{0}}<\infty$ for $a$ compact $K$-invariant neighbourhood $W \in \mathfrak{U}_{\mathbb{G}}$.

(c) $\eta$ has finite first-order moments (on $\mathbb{G}$ ), that is, $\int_{C U} f d \eta<\infty$ for all subadditive $f: \mathbb{G} \rightarrow \mathbb{R}_{+}$and $U \in \mathcal{U}_{\mathbb{G}}$.

(d) For some (any) $t_{0}>0, v\left(t_{0}\right)$ has finite logarithmic moments, that is, for any regular $\tau_{t_{0}}$-function $f$, one has $\int_{N} \log (1+f) \mathrm{d} \nu\left(t_{0}\right)<\infty$.

$\left(\mathrm{d}^{*}\right) \int_{N} \log (1+\phi) d \nu\left(t_{0}\right)=\int_{N} \log (1+\|x\|) d \nu\left(t_{0}\right)(x, \kappa)<$ $\infty$.

(e) $\eta$ (considered as measure on $N$ ) has finite logarithmic moments, that is, for all $t, \int_{N \backslash U} \log (1+f) d \eta<\infty$ for all regular $\tau_{t}$-functions, for all $U \in \mathfrak{U}_{N}$.

$\left(\mathrm{e}^{*}\right) \int_{N \backslash U} \log (1+\phi) d \eta=\int_{N \backslash U} \log (1+\|x\|) d \eta(x, \kappa)<$ $\infty$, for all $U \in \mathfrak{U}_{N}$.

Proof. According to Proposition 12 and Theorem 20 we know that condition (a) is equivalent with shift compactness of a skeleton random walk $\left\{\lambda_{t_{0}}^{k}\right\}$ for some (hence any) $t_{0}>0$. Hence (a) is equivalent to (b1) For some [any] $t_{0}>0$, for any subadditive function $f$ on $\mathbb{G}_{t_{0}}, \int_{\mathbb{G}_{t_{0}}} f \mathrm{~d} \lambda_{t_{0}}<\infty$ (i.e., $\lambda_{t_{0}}$ has finite first-order moments on $\mathbb{G}_{t_{0}}$.)

Obviously, "(b1) $\Rightarrow(\mathrm{b})$ ". By Fact 2, “(b1) $\Leftrightarrow(\mathrm{d})$ "; furthermore, by Step 3 (cf. Lemma A.3), " $(\mathrm{d}) \Leftrightarrow\left(\mathrm{d}^{*}\right)$ ” and “ $\left(\mathrm{e}^{*}\right) \Leftrightarrow(\mathrm{e})$ ”.

According to Step 5 (Lemma A.5), (b) $\Leftrightarrow \int_{\mathbb{G}} \delta_{W_{t_{0}}} \mathrm{~d} \lambda_{t_{0}}<$ $\infty$, and therefore $(\mathrm{b}) \Leftrightarrow\left(\mathrm{b}^{*}\right) \int_{\mathbb{G}} \delta_{W} \mathrm{~d} \lambda_{t_{0}}<\infty$ for any $t_{0}>0$, equivalently, for any $t_{0}, t_{1}>0, \int_{\mathbb{G}} \delta_{W_{t_{1}}} \mathrm{~d} \lambda_{t_{0}}<\infty$, (since $\delta_{W} \sim$ $\delta_{W_{t_{0}}} \sim \delta_{W_{t_{1}}}$ ). 【Let us mention that (b1) $\Rightarrow \int_{\mathbb{G}_{t_{0}}} \delta_{V_{t_{0}}} \mathrm{~d} \lambda_{t_{0}}<\infty$, and since $\delta_{W_{t_{0}}} \leq \delta_{V_{t_{0}}}$, we obtain again “(b1) $\Rightarrow(\mathrm{b})$ ". \ Applying Siebert's result, Fact 3 , to the subadditive function $\delta_{W_{t_{0}}}$ (resp., to $\delta_{W}$ for any $K$-invariant relatively compact symmetric $W \in$ $\left.\mathcal{U}_{\mathbb{G}}\right)$ yields “ $\left(\mathrm{b}^{*}\right) \Leftrightarrow(\mathrm{c})$ ”.

$\log \psi$ is subadditive- $\psi$ as in Step 4 (Lemma A.4)-hence again by Fact 3 we obtain (b) $\Rightarrow \int_{\mathbb{G}} \log \psi \mathrm{d} \lambda_{t_{0}}<\infty \Leftrightarrow$ $\int_{C U} \log \psi \mathrm{d} \eta<\infty$ for all $U \in \mathcal{U}_{\mathbb{G}}$.

But we have $\int_{\mathbb{G}} \log \psi \mathrm{d} \lambda_{t_{0}}=\int_{N} \log (1+\phi) \mathrm{d} \nu\left(t_{0}\right)<\infty$, hence $(\mathrm{b}) \Rightarrow\left(\mathrm{d}^{*}\right)$ and $\int_{\mathbb{G} \backslash U} \log \psi \mathrm{d} \eta=\int_{N \backslash U \cap N} \log (1+\phi) \mathrm{d} \eta$.
Hence the left integral is finite iff $\left(\mathrm{e}^{*}\right)$ holds, whence $\left(\mathrm{d}^{*}\right) \Leftrightarrow$ $\left(e^{*}\right)$.

The proof is complete: “ $(\mathrm{a}) \Leftrightarrow(\mathrm{b} 1)$ ", “ $(\mathrm{b} 1) \Rightarrow(\mathrm{b})$ ",

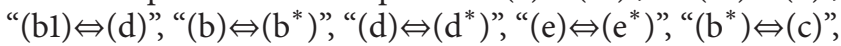
" $(\mathrm{b}) \Rightarrow\left(\mathrm{d}^{*}\right)$ ", and " $\left(\mathrm{d}^{*}\right) \Leftrightarrow\left(\mathrm{e}^{*}\right)$ ".

We close with two examples in analogy with the case of discrete times. The first shows that $\lim _{t \rightarrow \infty} \nu(t)$ does not need to exist if $N=C_{K}(T), K \neq\{e\}$. (For $K=\{e\}$, i.e., $N=C(T)$, $\lim _{t} \nu(t)$ always exists, cf. [22, Proposition A]).

Example 23. Let $\Gamma$ be a contractible Lie group, hence simply connected and nilpotent, with contracting one-parameter group $S=\left(\sigma_{t}\right)_{t>0}$ in $\operatorname{Aut}(\Gamma)$, and let $K$ be a solenoidal compact group with dense one-parameter subgroup $(z(t))_{t \in \mathbb{R}}$. Put $N:=\Gamma \otimes K$ and define $T:=\left(\tau_{t}\right) \subseteq \operatorname{Aut}(N)$ by $\tau_{t}=\sigma_{t} \otimes i d$, $t \in \mathbb{R}$. Hence $N=C_{K}(T)$. Let $\{\mu(t)\}$ be a continuous $M-$ semigroup (with respect to $S$ ) in $\mathscr{M}^{1}(\Gamma)$ such that $\rho=\lim \mu(t)$ exists. As $\xi_{t}:=\left.\tau_{t}\right|_{K} \equiv i d$, continuous M-semigroups in $\mathscr{M}^{1}(K)$ (with respect to $\left(\xi_{t}\right)$ ) are just continuous convolution semigroups; in particular, $\left\{\varepsilon_{z(t)}\right\}$ is a M-semigroup. It is immediately verified that $\left\{\nu(t):=\mu(t) \otimes \varepsilon_{z(t)}\right\}$ is a relatively compact M-semigroup in $\mathscr{M}^{1}(N)$ (with respect to $T$ ), with nondissipating continuous convolution semigroup $\left\{\lambda_{t}:=\right.$ $\left.\nu(t) \otimes \varepsilon_{t}\right\}$.

As $(z(t))$ is dense in $K, \operatorname{LIM}\{\nu(t): t \rightarrow \infty\}=\left\{\rho \otimes \varepsilon_{\kappa}:\right.$ $\kappa \in K\}$. In particular, $\lim _{t \rightarrow \infty} \nu(t)$ does not exist.

In analogy to Example 9, the next example shows connections between stable laws and nondissipating continuous convolution semigroups (cf., e.g., $[6, \$ 2.3]$ ).

Example 24. Stable laws are particular self-decomposable laws. To show this we have to switch between additive and multiplicative parametrizations of continuous one-parameter groups.

Let $N$ be a simply connected nilpotent Lie group and $S=\left(\sigma_{t}\right) \subseteq \operatorname{Aut}(N)$ be a contracting continuous group with multiplicative parametrization, $\sigma_{t} \sigma_{s}=\sigma_{t \cdot s}$ for $t, s>0$, and $\lim _{t \rightarrow 0} \sigma_{t}(x)=e$ for all $x \in N$. A continuous convolution semigroup $\left\{\rho_{t}\right\}$ in $\mathscr{M}^{1}(N)$ is called $S$-stable if $\sigma_{t}\left(\rho_{1}\right)=\rho_{t}$, for all $t>0$, equivalently, $\sigma_{t}\left(\rho_{s}\right)=\rho_{t \cdot s}$ for $t>0, s \geq 0$. Put $\rho:=\rho_{1}$. We have $\rho=\rho_{1-s} \star \rho_{s}=\rho_{1-s} \star \sigma_{s}(\rho)$, for all $0<s \leq 1$; hence $\rho$ is $\sigma_{s}$-decomposable with cofactors $\rho_{1-s} \in \operatorname{Cof}_{\rho}\left(\sigma_{s}\right)$.

To obtain a continuous $\mathrm{M}$-semigroup of cofactors we have to switch to additive parametrization: $T:=\left(\tau_{t}:=\sigma_{\mathrm{e}^{-t}}\right)_{t \in \mathbb{R}}$ is a continuous one-parameter group satisfying $\tau_{t+s}=\tau_{t} \tau_{s}$ and $\lim _{t \rightarrow \infty} \tau_{t}(x)=e$ for all $x \in N$. And with this notations we obtain $\rho=\rho_{1-\mathrm{e}^{-t}} \star \tau_{t}(\rho)$ for $t \geq 0$.

As immediately verified, $\left\{\nu(t):=\rho_{1-e^{-t}}\right\}_{t \in \mathbb{R}_{+}}$is a relatively compact continuous M-semigroup with $\nu(t) \in \operatorname{Cof}_{\rho}\left(\tau_{t}\right)$ (and $\rho=\lim \nu(t))$; hence it defines a nondissipating continuous convolution semigroup $\left\{\lambda_{t}=\rho_{1-e^{-t}} \otimes \varepsilon_{t}\right\} \subseteq \mathscr{M}^{1}(\mathbb{G}), \mathbb{G}=$ $N \rtimes_{T} \mathbb{R}$. Note that in this (trivial) case, the M-semigroup belongs to $\left\{\rho_{s}\right\}_{s \geq 0}$, respectively, to the orbit $\left\{\tau_{t}(\rho)\right\}_{t \geq 0}: \nu(t)=$ $\rho_{1-e^{-t}}=\sigma_{1-e^{-t}}(\rho)=\tau_{-\log \left(1-e^{-t}\right)}(\rho)$.

Analogously, stable laws with nontrivial idempotents on $N=C \rtimes_{T} K$ generate $T$-decomposable laws (see $[6, \S 3.5]$ ). 
A variety of examples of (self-) decomposable laws are obtained via subordination as in Example 11: Let $\left\{\rho_{t}\right\}$ be a continuous convolution semigroup which is stable with respect to $T$ as above. Let $\xi$ be a $\left(H_{e^{-t}}\right)$-decomposable probability measure on $\mathbb{R}_{+}$, where $H_{\alpha}: x \mapsto \alpha \cdot x$ denotes the homothetic transformation on $\mathbb{R}$. Then, as immediately verified, the subordinated measure $\mu:=\int_{0}^{\infty} \rho_{t} \mathrm{~d} \xi(t)$ is $T$-decomposable. In particular, if $\xi$ is an exponential distribution, we obtain geometric stable laws (or geometric semistable laws, if $\left\{\rho_{t}\right\}$ is semi stable, cf. $[6, \S 2.13$ resp., 2.14.36]. There the results are formulated for aperiodic groups, hence for M-semigroups with trivial idempotents. But the proofs do not depend on that assumption).

\section{Appendix}

\section{Group Norms and $\tau_{t}$-Functions}

Let, as before, $N$ be a locally compact group, $T=\left(\tau_{t}\right) \subseteq$ $\operatorname{Aut}(N)$, a continuous one-parameter group such that $N=$ $C_{K}(T)$.

Lemma A.1. There exists a homogeneous norm $\|\cdot\|$ on $C(T)$ which is in addition a $\beta(K)$-invariant.

Proof. Put, as before, $C=C(T) .\left.T\right|_{C}=\left(\left.\tau_{t}\right|_{C}\right)_{t \in \mathbb{R}}$ defines a group of dilations, that is, a multiplicative parametrized continuous group $D=\left(\delta_{s}\right)_{s>0} \subseteq \operatorname{Aut}(C), \delta_{s} \delta_{r}=\delta_{s r}$, and a subadditive function $\|\cdot\|_{1}: C \rightarrow \mathbb{R}_{+}$such that $\left\|x^{-1}\right\|_{1}=$ $\|x\|_{1},\|x\|_{1}=0$ iff $x=e$ and $\left\|\delta_{s}(x)\right\|_{1}=s\|x\|_{1}$ for all $s>0$, $x \in C$. Furthermore, $\delta_{s}$ commutes with all automorphisms $\alpha \in \operatorname{Aut}(C)$ which commute with all $\tau_{t}$.

【In fact, let $\mathbb{V}$ denote the Lie algebra of $C$. Then $\operatorname{Aut}(C) \cong$ $\operatorname{Aut}(\mathbb{V}) \subseteq \mathrm{GL}(\mathbb{V})$, and the correspondence is expressed by $\operatorname{Aut}(C) \ni \alpha \leftrightarrow \stackrel{\circ}{\alpha} \in \operatorname{Aut}(\mathbb{V}) . \stackrel{\circ}{\delta}_{s}$ can be defined via the Jordan decomposition of the exponent E, defined by $\dot{\tau}_{t}=e^{t E}$ (cf. [29, Proof of Proposition 2.4], [6, 2.1.13]). Whence the assertion follows.]

As by assumption $\left(\left.\tau_{t}\right|_{C}\right) \subseteq \operatorname{Cent}(\beta(K)$, Aut $(C))$ (Proposition 17) it follows that $\left(\delta_{s}\right) \subseteq \operatorname{Cent}(\beta(K)$, Aut $(C)$ ).

Put $\|x\|:=\int_{K}\|\beta(\kappa)(x)\|_{1} \mathrm{~d} \omega_{K}(\kappa)$, with $\omega_{K}$ denoting the Haar measure. As immediately seen, $\|\cdot\|$ is symmetric, subadditive, with $\|x\|=0$ iff $x=e,\{\|x\| \leq \alpha\}$ compact in $\mathfrak{U}_{C}$ for $\alpha>0$, and $\|\cdot\|$ is $\beta(K)$-invariant by construction. Furthermore, $\left\|\delta_{s}(x)\right\|=\int_{K}\left\|\beta(\kappa)\left(\delta_{s}(x)\right)\right\|_{1} \mathrm{~d} \omega_{K}(\kappa)=$ $\int_{K}\left\|\delta_{s}(\beta(\kappa)(x))\right\|_{1} \mathrm{~d} \omega_{K}(\kappa)=s \int_{K}\|\beta(\kappa)(x)\|_{1} \mathrm{~d} \omega_{K}(x)=$ $s\|x\|$.

Lemma A.2. Define the function $\phi: N=C \rtimes_{\beta} K \rightarrow \mathbb{R}_{+}$by $\phi(x, \kappa):=\|x\|$. Then $\phi$ is subadditive and continuous, satisfies $\phi(\gamma)=\phi\left(\gamma^{-1}\right), \phi(\gamma)=0$ iff $\gamma=(e, \kappa) \in K$, and furthermore, $\phi$ is $K$-bi-invariant.

Moreover, for any $t_{0}>0$ there exist constants $0<r \leq R<$ $1,0<s \leq S<1$, satisfying for all $\gamma \in N$ the growth conditions

$$
\begin{array}{ll}
r^{n} \phi(\gamma) \leq \phi\left(\tau_{t_{0}}^{n}(\gamma)\right) \leq R^{n} \phi(\gamma), & n \geq 0, \\
S^{n} \phi(\gamma) \leq \phi\left(\tau_{t_{0}}^{n}(\gamma)\right) \leq s^{n} \phi(\gamma), & n<0 .
\end{array}
$$

For large $t_{0}$ one can put for example $R:=\left\|\left.\tau_{t_{0}}\right|_{C}\right\|, r:=$ $\left\|\left.\tau_{t_{0}}^{-1}\right|_{C}\right\|, S=R, s=r$. The automorphism norm is defined as $\|\sigma\|:=\sup \{\|\sigma(x)\|: x \in C,\|x\|=1\}$. In particular, one has $\left\|\left.\tau_{s+t}\right|_{C}\right\| \leq\left\|\left.\tau_{t}\right|_{C}\right\| \cdot\left\|\left.\tau_{s}\right|_{C}\right\|,\left\|\left.\tau_{s}\right|_{C}\right\| \rightarrow 0$ for $t \rightarrow \infty$ and $\left\|\left.\tau_{-t}\right|_{C}\right\| \geq 1$ for $t \geq 0$

Proof. Obviously, $\phi$ is continuous, $\phi \geq 0, \phi(x, \kappa)=0$ iff $x=$ $e$, that is, iff $(x, \kappa) \in K$. $\phi$ is subadditive: $\phi\left((x, \kappa)\left(y, \kappa^{\prime}\right)\right)=$ $\phi\left(x \beta(\kappa)(y), \kappa \kappa^{\prime}\right)=\|x \beta(\kappa)(y)\| \leq\|x\|+\|\beta(\kappa)(y)\|=\|x\|+$ $\|y\|=\phi(x, \kappa)+\phi\left(y, \kappa^{\prime}\right)$.

According to $[29$, Proposition 2.5(a) and (3.1)], [6, 2.10.15(b) and (2.14.11)], there exist $s, r, S, R$ satisfying the growth conditions (A.1) for $C,\|\cdot\|$ and $\left.\tau_{t}\right|_{C} \in \operatorname{Aut}(C)$. (We

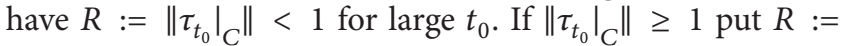
$\left\|\left.\tau_{m t_{0}}\right|_{C}\right\|^{1 / m}<1$ for sufficiently large $m$.)

Thus by the definition of $\phi$ the growth conditions (A.1) for $N$ follow.

Finally, $\phi\left(\left(e, \kappa_{1}\right)\left(x, \kappa_{0}\right)\left(e, \kappa_{2}\right)\right)=\phi\left(\beta\left(\kappa_{1}\right)(x), \kappa_{1} \kappa_{0} \kappa_{2}\right)=$ $\phi\left(x, \kappa_{0}\right)$ for $x \in N, \kappa_{i} \in K$ shows $K$-bi-invariance of the pseudo-group norm $\phi$.

Lemma A.3. Let $f: N \rightarrow \mathbb{R}_{+}$be a regular $\tau_{t_{0}}$-function for some $t_{0}>0, f\left(\tau_{t_{0}}^{p}(\gamma)\right)=\alpha f(\gamma)$ (for some $\alpha>0$ ) (cf. Definition 1).

Then one obtains $\log (1+f) \sim \log (1+\phi)$, ( $\phi$ defined in Lemma A.2).

Proof (similar to proof of Lemma 2.9 in [1]). With $t_{1}=p t_{0}>$ 0 we have $f\left(\tau_{t_{1}}(\gamma)\right)=\alpha f(\gamma)$. Put $C_{0}:=\{f \leq 1\}, C_{n}:=$ $\tau_{t_{1}}^{-n}\left(C_{0}\right), n \in \mathbb{Z}$. Hence $n \mapsto C_{n}$ is increasing and $N=$ $K \cup \bigcup_{n \in \mathbb{Z}}\left(C_{n+1} \backslash C_{n}\right) . f$ is a regular $\tau_{t_{1}}$-function and hence $C_{0}$, and thus all $C_{n}$ are compact neighbourhoods in $\mathfrak{U}_{N}$, and by definition of $f, C_{n}=\left\{f \leq \alpha^{-n}\right\}$. $\phi$ is continuous and subadditive, hence locally bounded. Thus there exist constants $m, M$, such that $0 \leq m \leq \phi(\gamma) \leq M<\infty$ for all $\gamma \in C_{0} \backslash C_{-1}$. According to Lemma A.2, we obtain for $\gamma \in C_{n} \backslash C_{n-1}$, hence for $\tau_{t_{1}}^{n}(\gamma) \in C_{0} \backslash C_{-1}$ :

$$
\begin{array}{ll}
S^{-n} m \leq \phi(\gamma)=\phi\left(\tau_{t_{1}}^{-(n+1)} \tau_{t_{1}}^{n+1}(\gamma)\right) \leq s^{-n} M, & n \geq 0, \\
r^{-n} m \leq \phi(\gamma)=\phi\left(\tau_{t_{1}}^{-(n+1)} \tau_{t_{1}}^{n+1}(\gamma)\right) \leq R^{-n} M, & n<0 .
\end{array}
$$

On the other hand, for $n \geq 0, \alpha^{-n} \leq f(\gamma) \leq \alpha^{-(n+1)}$, equivalently, $n+1 \geq \log f(\gamma)^{-1 / \log (\alpha)} \geq n$. Inserting this in (A.2) yields

$$
m f(\gamma)^{\log S / \log \alpha} \leq \phi(\gamma) \leq M f(\gamma)^{\log s / \log \alpha}, \quad n \geq 0
$$

and analogous estimates for $n<0$.

Together, there exist constants $u, v, U, V$ such that $u f^{v} \leq$ $\phi \leq U f^{V}$, whence $\log (1+\phi) \sim \log (1+f)$ follows.

Lemma A.4. With the notations of the preceding lemmas define $\psi: \mathbb{G}=N \rtimes_{T} \mathbb{R} \rightarrow \mathbb{R}_{+}$by $\psi(\gamma, t):=1+\phi(\gamma)+\left\|\left.\tau_{-|t|}\right|_{C}\right\|$.

Then $\psi$ is continuous, $\geq 2$, submultiplicative and satisfies $\psi(\gamma, t)=2$ iff $t=0, \gamma \in K$. Therefore, $\log \psi$ is subadditive 
and $>0$. Moreover $\{\psi<\alpha\}$ is relatively compact and belongs to $\mathfrak{U}_{\mathbb{G}}$ for all $\alpha>2$.

Proof (compare with Propositions 4.6 in [29], 2.14.28 in [6]). For short, we write $\left\|\tau_{t}\right\|$ instead of $\left\|\left.\tau_{t}\right|_{C}\right\|$. We have $\left\|\tau_{t}\right\| \stackrel{t \rightarrow \infty}{\longrightarrow} 0$.

The automorphism norm, restricted to $\operatorname{Cent}(\operatorname{Aut}(C)$, $\left(\delta_{t}\right)$ ), is submultiplicative, thus $\left\|\tau_{t+s}\right\| \leq\left\|\tau_{t}\right\| \cdot\left\|\tau_{s}\right\|$, satisfies $\left\|\tau_{t}\right\| \leq\left\|\tau_{-|t|}\right\|,\left\|\tau_{-|t|}\right\| \geq 1,\left\|\tau_{0}\right\|=1$, and we have $\phi\left(\tau_{t}(\gamma)\right) \leq$ $\left\|\tau_{-|t|}\right\| \cdot \phi(\gamma)$. Hence

$$
\begin{aligned}
\psi & \left((\gamma, t)\left(\gamma^{\prime}, s\right)\right) \\
& =\psi\left(\gamma \tau_{t}\left(\gamma^{\prime}\right), t+s\right) \\
& =1+\phi\left(\gamma \tau_{t}\left(\gamma^{\prime}\right)\right)+\left\|\tau_{-|t+s|}\right\| \\
& \leq 1+\phi(\gamma)+\phi\left(\tau_{t}\left(\gamma^{\prime}\right)\right)+\left\|\tau_{-|t|}\right\| \cdot\left\|\tau_{-|s|}\right\| \\
& \leq 1+\phi(\gamma)+\left\|\tau_{-|t|}\right\| \cdot \phi\left(\gamma^{\prime}\right)+\left\|\tau_{-|t|}\right\| \cdot\left\|\tau_{-|s|}\right\| \\
& \leq\left(1+\phi(\gamma)+\left\|\tau_{-|t|}\right\|\right) \cdot\left(1+\phi\left(\gamma^{\prime}\right)+\left\|\tau_{-|s|}\right\|\right) .
\end{aligned}
$$

Whence submultiplicativity of $\psi$ follows. Furthermore, $\psi(\gamma, t)=1+\phi(\gamma)+\left\|\tau_{-|t|}\right\| \geq 2+\phi(\gamma) \geq 2$, and $\psi(\gamma, t)=2$ iff $t=0$ and $\phi(\gamma)=0$, that is, iff $(\gamma, t) \in K$. The last assertion follows since $\psi(\gamma, t)<\alpha \Leftrightarrow \phi(\gamma)+\left\|\tau_{-|t|}\right\|<\alpha-1$.

Lemma A.5. Let, as before, $N=C \rtimes_{\beta} K$ and $\mathbb{G}=N \rtimes_{T} \mathbb{R} . N$ is compactly generated: for any symmetric $U \in \mathcal{U}_{C}, V:=U \cdot K$ generates $N$, and, for example, for any $\epsilon>0, W:=V \otimes[-\epsilon, \epsilon]$ generates $\mathbb{G}$. Let $f$ be a regular $\tau_{t_{0}}$-function, for some $t_{0}>0$. Let, for $t>0, W_{t}:=\bigcup_{0 \leq s \leq t}\left\{V \otimes\{s\} \cup\{V \otimes\{s\}\}^{-1}\right\}$, where $V=\{f \leq 1\}$.

Then $W_{t}$ generates $\mathbb{G}$ and for any symmetric relatively compact $W \in \mathfrak{U}_{\mathbb{G}}$ with $W=W K$, one has $\delta_{W_{t}} \sim \delta_{W}$. In particular, for all $t_{1}, t_{2}>0$ it follows that $\delta_{W_{t_{1}}} \sim \delta_{W_{t_{2}}}$.

Proof. Let again $T=\left(\tau_{t}=\left.i_{x(t)}\right|_{N}\right)$. By assumption, $(x(t))$ and $K$ commute element wise. We have $N=C \cdot K, C \triangleleft N, K \subseteq$ $N, C \cap K=\{e\}$. Put $X:=(x(t)) \subseteq \mathbb{G}$, then $\Gamma:=C \cdot X \cong C \rtimes_{\left.T\right|_{C}} \mathbb{R}$ is a subgroup of $\mathbb{G}$, such that $\Gamma K=C X K=C K X=\mathbb{G}$. Hence $\mathbb{G} \cong N \rtimes_{T} \mathbb{R} \cong\left(C \rtimes_{\left.T\right|_{C}} \mathbb{R}\right) \rtimes_{\bar{\beta}} K$, where the action $\bar{\beta}$ is trivial on $\mathbb{R}$, that is, $\bar{\beta}(\kappa)(c, t)=(\beta(\kappa)(c), t)$.

$\Gamma$ is connected, hence any neighbourhood $B \in \mathfrak{U}_{\Gamma}$ generates $\Gamma$. Thus $B \cdot K$ generates $\mathbb{G}$.

The resting assertions follow immediately since $W_{t_{0}} \in$ $\mathfrak{U}_{\mathbb{G}}, W_{t_{0}} K=W_{t_{0}}$ (since $f$ is $K$-bi-invariant, cf. [1, Proposition $2.8]$ ). Hence according to [28, Remarque 3], $\delta_{W_{t_{0}}} \sim \delta_{B} \sim \delta_{W_{t_{1}}}$ for any $t_{0}, t_{1}>0$.

\section{References}

[1] W. Jaworski, "On shifted convolution powers and concentration functions in locally compact groups," in Probability on Algebraic Structures: AMS Special Session on Probability on Algebraic Structures, March 12-13, 1999, Gainesville, Florida, USA, vol. 261 of Contemporary Mathematics, pp. 23-41, 2000.
[2] E. Siebert, "Contractive automorphisms on locally compact groups," Mathematische Zeitschrift, vol. 191, no. 1, pp. 73-90, 1986.

[3] E. Siebert, "Semistable convolution semigroups and the topology of contraction groups," in Probability Measures on Groups IX: Proceedings of a Conference held in Oberwolfach, FRG, January 17-23, 1988, H. Heyer, Ed., vol. 1379 of Lecture Notes in Mathematics, pp. 325-343, 1989.

[4] W. Hazod and E. Siebert, "Automorphisms on a Lie group contracting modulo a compact subgroup and applications to semistable convolution semigroups," Journal of Theoretical Probability, vol. 1, no. 2, pp. 211-225, 1988.

[5] W. Hazod and E. Siebert, "Continuous automorphism groups on a locally compact group contracting modulo a compact subgroup and applications to stable convolution semigroups," Semigroup Forum, vol. 33, no. 1, pp. 111-143, 1986.

[6] W. Hazod and E. Siebert, Stable Probability Measures on Euclidean Spaces and on Locally Compact Groups. Structural Properties and Limit Theorems, vol. 531 of Mathematics and its Applications, Kluwer Academic, New York, NY, USA, 2001.

[7] R. Shah, "Semistable measures and limit theorems on real and p-adic groups," Monatshefte für Mathematik, vol. 115, no. 3, pp. 191-213, 1993.

[8] R. Shah, "Selfdecomposable measures on simply connected nilpotent groups," Journal of Theoretical Probability, vol. 13, no. 1, pp. 65-83, 2000.

[9] S. G. Dani and R. Shah, "Collapsible probability measures and concentration functions on Lie groups," Mathematical Proceedings of the Cambridge Philosophical Society, vol. 122, no. 1, pp. 105-113, 1997.

[10] S. G. Dani and R. Shah, "Contraction subgroups and semistable measures on $p$-adic Lie groups," Mathematical Proceedings of the Cambridge Philosophical Society, vol. 110, no. 2, pp. 299-306, 1991.

[11] W. Jaworski, "On contraction groups of automorphisms of totally disconnected locally compact groups," Israel Journal of Mathematics, vol. 172, pp. 1-8, 2009.

[12] W. Jaworski, "Contractive automorphisms of locally compact groups and the concentration function problem," Journal of Theoretical Probability, vol. 10, no. 4, pp. 967-989, 1997.

[13] W. Jaworski, J. Rosenblatt, and G. Willis, "Concentration functions in locally compact groups," Mathematische Annalen, vol. 305, no. 4, pp. 673-691, 1996.

[14] H. Glöckner, "Contraction groups for tidy automorphisms of totally disconnected groups," Glasgow Mathematical Journal, vol. 47, no. 2, pp. 329-333, 2005.

[15] U. Baumgartner and G. A. Willis, "Contraction groups and scales of automorphisms of totally disconnected locally compact groups," Israel Journal of Mathematics, vol. 142, pp. 221-248, 2004.

[16] I. Csiszár, “On infinite products of random elements and infinite convolutions of probability distributions on locally compact groups," Zeitschrift für Wahrscheinlichkeitstheorie und Verwandte Gebiete, vol. 5, pp. 279-295, 1966.

[17] P. Bougerol, "Fonctions de concentration sur certains groupes localement compacts," Zeitschrift für Wahrscheinlichkeitstheorie und Verwandte Gebiete, vol. 45, no. 2, pp. 135-157, 1978.

[18] K. H. Hofmann and A. Mukherjea, "Concentration functions and a class of noncompact groups," Mathematische Annalen, vol. 256, no. 4, pp. 535-548, 1981. 
[19] A. Tortrat, "Convolutions dénombrables équitendues dans un groupe topologique $X$," in Les Probabilités sur les Structures Algébriques, Editions du CNRSaris, Paris, France, 1970.

[20] A. Tortrat, "Lois tendues et convolutions dénombrables dans un groupe topologique X," Annales de l'Institut Henri Poincaré B, vol. 2, pp. 279-298, 1966.

[21] K. H. Hofmann and Z. J. Jurek, "Some analytical semigroups occurring in probability theory," Journal of Theoretical Probability, vol. 9, no. 3, pp. 745-763, 1996.

[22] W. Hazod, "On Mehler semigroups, stable hemigroups and selfdecomposability," in Infinite Dimensional Harmonic Analysis III. Proceedings Tübingen, H. Heyer, T. Hirai, T. Kawazoe, and K. Saito, Eds., pp. 83-98, World Scientific, 2005.

[23] W. Hazod, "On some convolution semi- and hemigroups appearing as limit distributions of normalized products of group-valued random variables," in Analysis on Infinite Dimensional Lie groups and Algebras: Proceedings of the International Colloquium Cirm, Marseille, France 15-19 September, 1997, H. Heyer and J. Marion, Eds., pp. 104-121, 1998.

[24] P. Becker-Kern and W. Hazod, "Mehler hemigroups and embedding of discrete skew convolution semigroups on simply connected nilpotent Lie groups," in Infinite Dimensional Harmonic Analysis IV, Proccedings Tokyo, J. Hilgert, A. Hora, T. Kawazoe, N. Nishiyama, and M. Voit, Eds., pp. 32-46, World Scientific, 2009.

[25] Y. Derriennic and M. Lin, "Convergence of iterates of averages of certain operator representations and of convolution powers," Journal of Functional Analysis, vol. 85, no. 1, pp. 86-102, 1989.

[26] P. Eisele, Zur Konvergenz transformierter Folgen von Faltungspotenzen eines Wahrscheinlichkeitsmaßes auf einer topologischen Gruppe [Dissertation], Universität Tübingen, 1991.

[27] P. Eisele, "On shifted convolution powers of a probability measure," Mathematische Zeitschrift, vol. 211, no. 4, pp. 557-574, 1992.

[28] Y. Guivarc'h, "Sur la loi des grands nombres et le rayon spectral d'une marche aléatoire," Asterisque, vol. 74, pp. 47-98, 1980.

[29] W. Hazod and H.-P. Scheffler, "Strongly $\tau$-decomposable and selfdecomposable laws on simply connected nilpotent Lie groups," Monatshefte für Mathematik, vol. 128, no. 4, pp. 269282, 1999.

[30] W. Hazod and K. Kosfeld, "Multiple decomposability of probabilities on contractible locally compact groups," Proceedings of the Indian Academy of Sciences-Mathematical Science, vol. 123, pp. 257-281, 2013.

[31] O. K. Zakusilo, "Some properties of random vectors of the form $\sum_{i=0}^{\infty} A^{i} \xi_{i}$," Teorija Verojatnostě̆ $i$ Matematičeskaja Statistika, vol. 13, pp. 59-52, 1975 (Russian), English tranlation Theory of Probability and Mathematical Statistics, vol. 13, pp. 62-64, 1977.

[32] Z. J. Jurek and J. D. Mason, Operator Limit Distributions in Probability Theory, John Wiley \& Sons, New York, NY, USA, 1993.

[33] N. Bourbaki, Eléments de Mathématique. Livre VI: Integration, chapter 7, Hermann, Paris, France, 1963.

[34] M. Tkačenko, "Introduction to topological groups," Topology and its Applications, vol. 86, no. 3, pp. 179-231, 1998.

[35] K. R. Parthasarathy, Probability Measures on Metric Spaces, Academic Press, New York, NY, USA, 1967.

[36] H. Heyer, Probability Measures on Locally Compact Groups, Springer, Berlin, Germany, 1977.

[37] W. Hazod and Y. Khohlov, "On Szasz's compactness theorem and applications to geometric stability," Probability and Mathematical Statistics, vol. 16, pp. 143-146, 1996.
[38] J. S. P. Wang, “The Mautner phenomenon for p-adic Lie groups," Mathematische Zeitschrift, vol. 185, no. 3, pp. 403-412, 1984.

[39] W. Jaworski, "Contraction groups, ergodicity, and distal properties of automorphisms of compact groups," Illinois Journal of Mathematics. In press.

[40] C. R. E. Raja and R. Shah, "Some properties of distal actions on locally compact groups," To be published.

[41] R. K. Lashof, "Lie algebras of locally compact groups," Pacific Journal of Mathematics, vol. 7, pp. 1145-1162, 1957.

[42] K. H. Hofmann and P. Mostert, "Splitting in topological groups," Memoirs of the American Mathematical Society, vol. 43, pp. 1-75, 1963.

[43] K. H. Hofmann, "Locally compact semigroups in which a subgroup with compact complement is dense," Transactions of the American Mathematical Society, vol. 106, pp. 19-51, 1963.

[44] E. Siebert, "Einbettung unendlich teilbarer Wahrscheinlichkeitsmaße auf topologischen Gruppen," Zeitschrift für Wahrscheinlichkeitstheorie und verwandte Gebiete, vol. 28, pp. 227-247, 1974.

[45] E. Siebert, "Continuous convolution semigroups integrating a submultiplicative function," Manuscripta Mathematica, vol. 37, no. 3, pp. 383-391, 1982.

[46] E. Siebert, "Jumps of stochastic processes with values in a topological group," Probability and Mathematical Statistics, vol. 5, no. 2, pp. 197-209, 1985. 


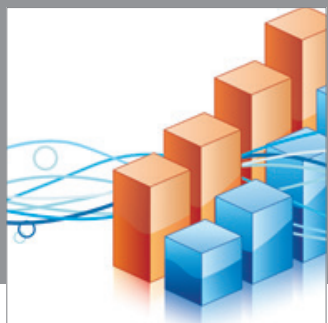

Advances in

Operations Research

mansans

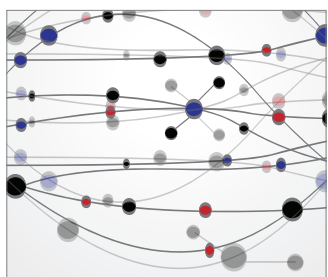

The Scientific World Journal
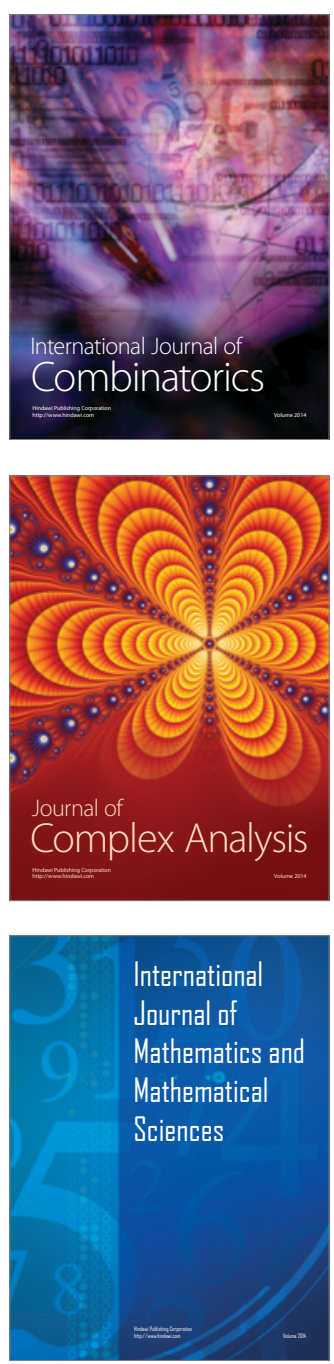
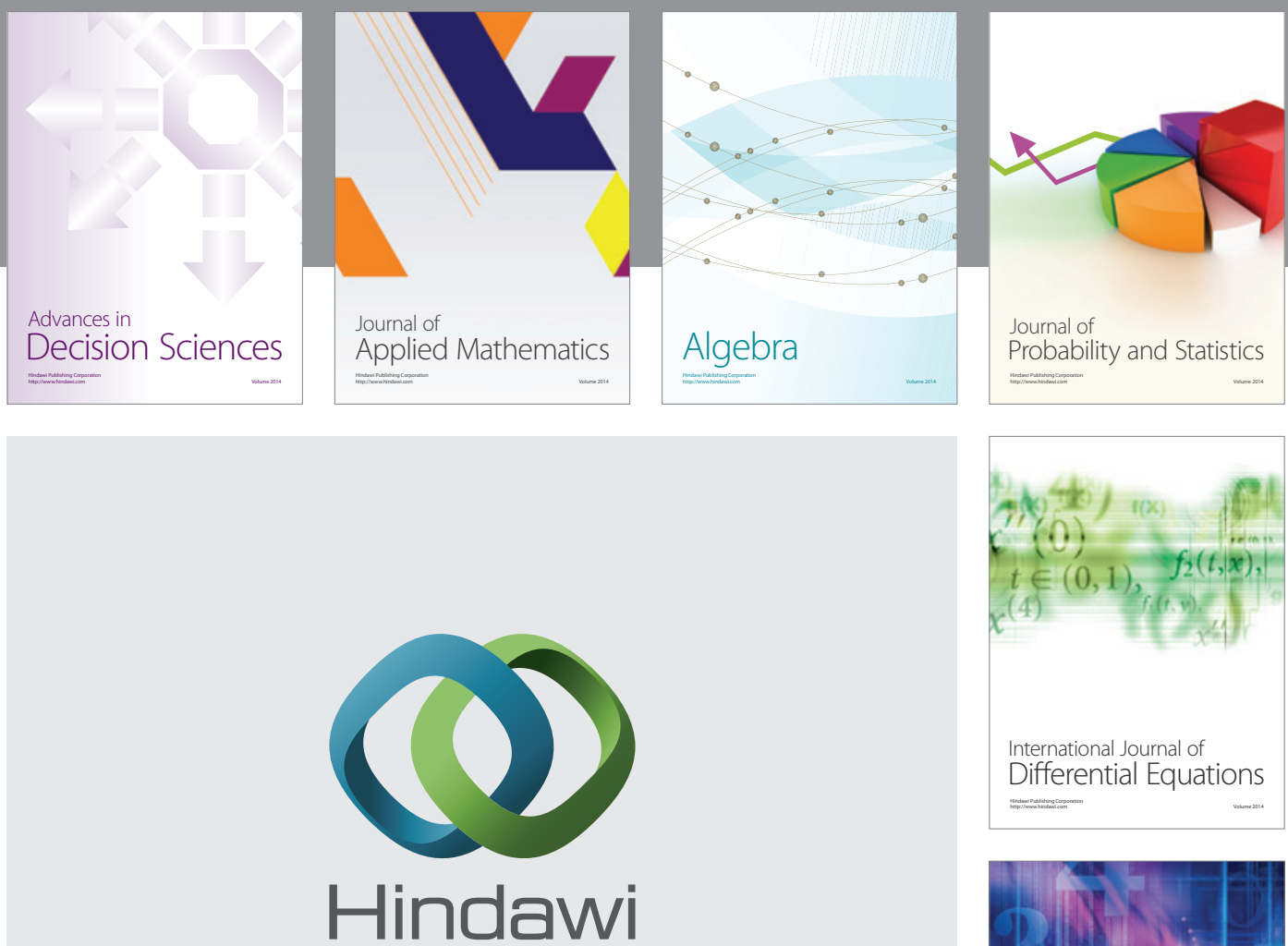

Submit your manuscripts at http://www.hindawi.com
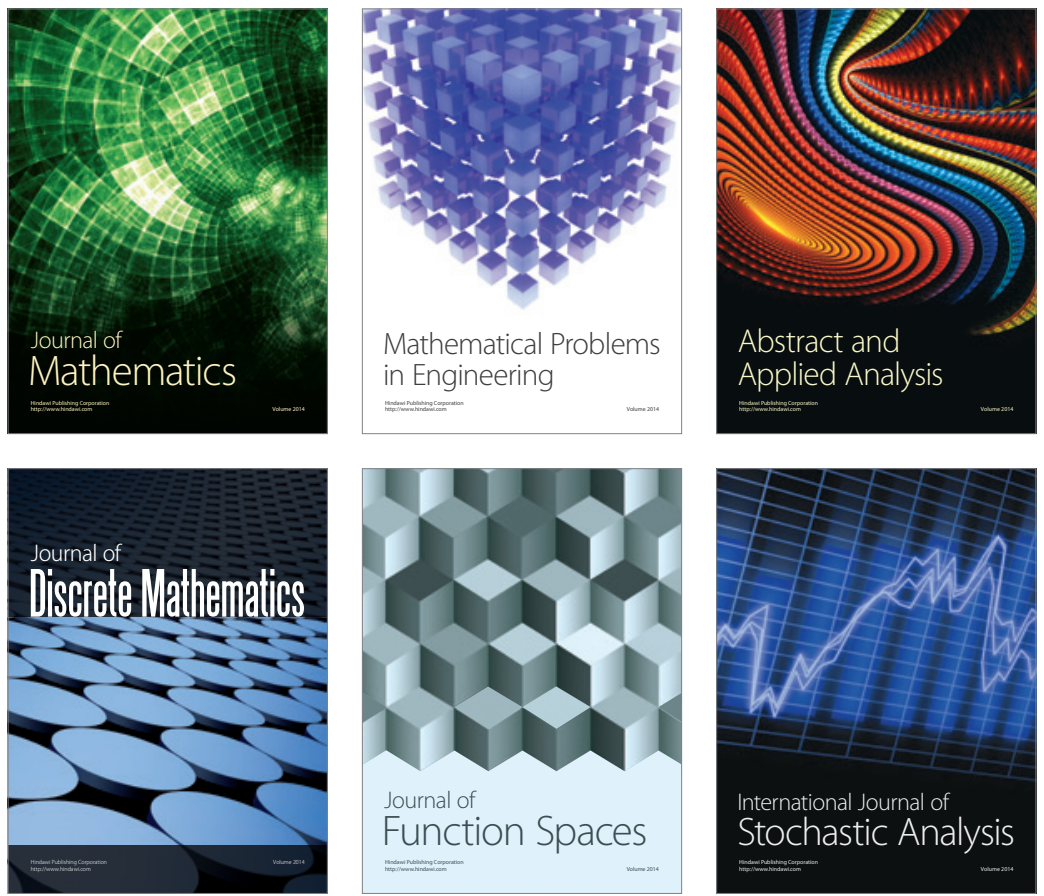

Journal of

Function Spaces

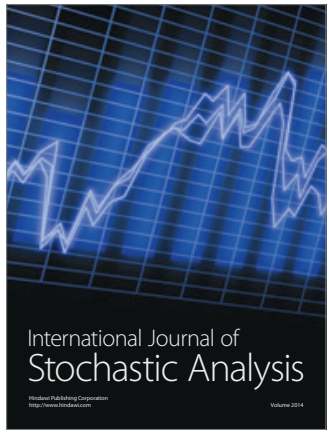

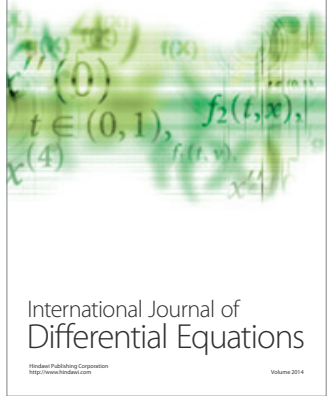
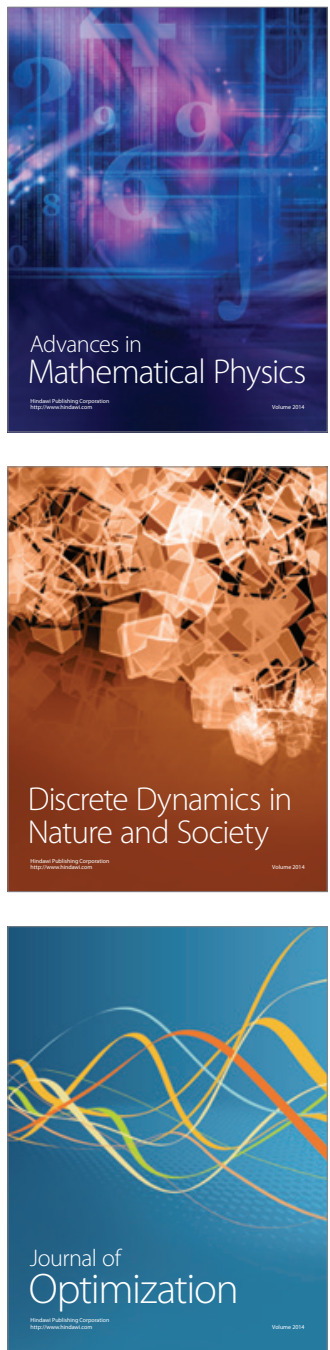